\title{
Combination Cancer Immunotherapy of Nanoparticle-Based Immunogenic Cell Death Inducers and Immune Checkpoint Inhibitors
}

This article was published in the following Dove Press journal: International Journal of Nanomedicine

Jing Qi

Feiyang Jin

Xiaoling $\mathrm{Xu}$

Yongzhong Du

Institute of Pharmaceutics, College of Pharmaceutical Sciences, Zhejiang University, Hangzhou, 310058, People's Republic of China
Correspondence: Yongzhong Du 866 Yu-Hang-Tang Road, Hangzhou, 310058 , People's Republic of China Tel +86-57I-88208435

Email duyongzhong@zju.edu.cn

\begin{abstract}
Cancer immunotherapy is a promising treatment strategy that aims to strengthen immune responses against cancer. However, the low immunogenicity of tumor cells and inhibition of effector $\mathrm{T}$ cells in the tumor immunosuppressive microenvironment remain two major challenges. Immunogenic cell death (ICD) inducers not only directly kill cancer cells but also increase the tumor immunogenicity and induce antitumor immune responses. Immune checkpoint inhibitors can alleviate the inhibition of immune cells. Significantly, the combination of ICD inducers and immune checkpoint inhibitors elicits a remarkable antitumor effect. Nanoparticles confer the ability to modulate systemic biodistribution and achieve targeted accumulation of administered therapeutic agents, thereby facilitating the clinical translation of immunotherapies based on ICD inducers in a safe and effective manner. In this review, we summarize the nanoparticle-based chemical and physical cues that induce effective tumor ICD and elicit an antitumor immune response. In particular, combination of ICD inducers with immune checkpoint inhibitors can further reverse immunosuppression and prevent tumor metastasis and recurrence. An overview of the future challenges and prospects is also provided.
\end{abstract}

Keywords: tumor immunotherapy, immunogenic cell death, nanoparticles, immune checkpoint inhibitors

\section{Introduction}

Cancer is one of the leading causes of death worldwide, and its treatment remains a formidable challenge. ${ }^{1}$ Traditional therapies such as surgery, chemotherapy, and radiotherapy are still standard treatment modalities and are used to achieve regression of the local tumor and improve patient survival in clinical practice. However, the poor prognosis, including cancer metastasis and recurrence, limits the application of these therapies. ${ }^{2}$ In recent years, cancer immunotherapy has developed as an additional treatment regimen, particularly in the areas of chimeric antigen receptor T cell therapy ${ }^{3}$ and immune checkpoint blockade therapy, ${ }^{4}$ which increase the strength of immune responses against cancer by either stimulating activities of the immune system or blocking signals produced by cancer cells to suppress immune responses. ${ }^{5}$ Cancer immunotherapy is a revolutionary treatment that aims to eliminate both local and distant metastatic tumors, and further elicit long-term immune memory to resist cancer recurrence; thus, it has been considered the most promising treatment for cancer. ${ }^{6}$ 
However, cancer cells are able to evade immune surveillance by down-regulating surface antigens to reduce immunogenicity and by inducing expression of immunosuppressive molecules to inhibit effector T cell activity. ${ }^{7,8}$ The low immunogenicity of tumor cells will interfere with the recognition and uptake by immune cells. Fortunately, some specific physical methods (eg, photodynamic therapy (PDT) and photothermal therapy (PTT)) and chemical drugs (eg, anthracyclines and oxaliplatin (OXA)) have been found to induce tumor cell death in an immunogenic manner. Immunogenic cell death (ICD) is characterized by release of tumor antigens, damage-associated molecular patterns (DAMPs), and pro-inflammatory cytokines, which facilitates the uptake and presentation of tumor antigens by immune cells, ultimately eliciting an antigenspecific antitumor immune response. ${ }^{9}$ Besides, the inhibition of effector $\mathrm{T}$ cells in tumor sites provides conditions for severe deterioration and spread of tumors. Importantly, immune checkpoint blockade immunotherapy has been applied to relieve immunosuppression and restore the antitumor activity of immune cells, and has been demonstrated to be effective for a variety of cancers. ${ }^{10-12}$ The most used immune checkpoints include: programmed death-1/ programmed death ligand-1 (PD-1/PD-L1), cytotoxic $\mathrm{T}$ lymphocyte-associated antigen-4 (CTLA-4), indoleamine 2.3-dioxygenase (IDO), and CD47. The combined application of ICD inducers and immune checkpoint inhibitors will be an effective strategy to suppress tumors and activate antitumor immune responses (Figure 1).

Despite advances in the development of combinations of ICD inducers and immune checkpoint inhibitors, there remain some challenges related to their clinical translation. ICD inducers lack tumor targeting ability; hence, their side effects and unsatisfactory efficacy tend to hinder their clinical applications. Nanoparticles have great potential for use in cancer treatments, with enhanced therapeutic efficacy and reduced side effects, mainly owing to their ability to modulate the systemic biodistribution and targeted accumulation of the administered therapeutic agents. ${ }^{13-15}$ Significantly, a better tumor treatment effect can be achieved when nanoparticle-based ICD inducers are applied with immune checkpoint inhibitors.

In this review, we summarize the features of tumor ICD and recent progress in the development of nanoparticle-based ICD inducers for combination cancer immunotherapy. Nanoparticle-based chemical or physical ICD

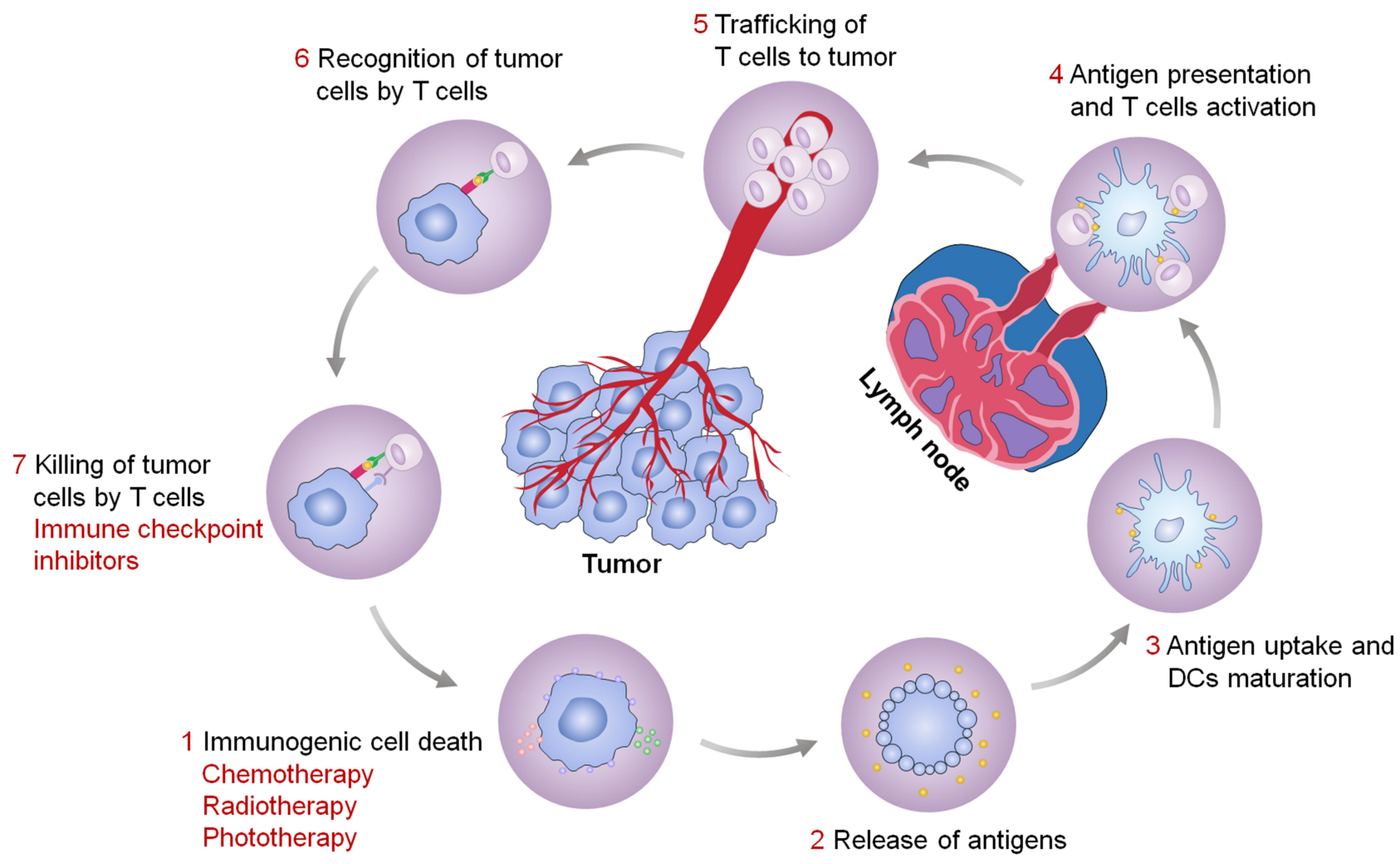

Figure I Antitumor immunity elicited by the treatment strategy of ICD inducers combined with immune checkpoint inhibitors. 
inducers are superior to free drugs in terms of antitumor efficiency. In particular, we provide an overview of the combination of nanoparticle-based ICD inducers and immune checkpoint inhibitors, which shows great potential for relieving tumor immunosuppression, promoting antitumor response, and preventing tumor metastasis and recurrence. Finally, we give a brief summary and discussion of future challenges and perspectives.

\section{Immunogenic Cell Death Features of ICD}

Over the past few years, the concept of ICD has emerged. As the name suggests, ICD is a process by which apoptotic cells can increase their immunogenicity and be recognized by various immune cells. ${ }^{16,17}$ Not all cell death results in immune activation - normal tissue has a rapid rate of cell turnover that does not provoke an inflammatory response. However, the death of only a few cells with increased antigenicity, especially tumor cells, can trigger a robust antigen-specific immune response. ${ }^{18}$

Cancer cells treated with anthracyclines, OXA, $\gamma$ irradiation, PDT, or PTT usually undergo ICD, accompanied by the release or exposure of molecules that can function as either adjuvant or danger signals for the innate immune system. ${ }^{19,20}$ These signals were later called DAMPs. ${ }^{21}$ Some DAMPs, including adenosine triphosphate (ATP) and high mobility group protein B1 (HMGB1), are secreted or released by cancer cells; others, including calreticulin (CRT) and heat shock protein 90, are exposed de novo or become enriched on the outer leaflet of the plasma membrane. Most of these molecules have predominantly immunological functions after their exposure on the cell surface or their secretion. ${ }^{20,22}$ Owing to interaction with phagocytosis receptors (purinergic receptors and pattern-recognition receptors, respectively), DAMPs are required for ICD, which ultimately leads to the activation of potent anticancer immunity. ${ }^{23-28}$ Moreover, the release of tumor-associated antigens and pro-inflammatory cytokines (such as IL-8, IL6 , IFN- $\beta$, and CXCL10) ${ }^{29}$ is also important during tumor ICD and can further facilitate the recognition of tumor cells by immune cells. Therefore, it is of great significance to study whether ICD occurs during tumor treatment.

\section{Mechanisms of ICD CRT Exposure}

CRT is the most abundant protein of the endoplasmic reticulum (ER) lumen, yet it can be found in other subcellular compartments, including the cytosol. $^{30,31}$ When cancer cells are exposed to ICD inducers, a fraction of CRT will translocate from the ER lumen to the surfaces of stressed and dying cells. ${ }^{23,32}$ This phenomenon might be due to the ER stress response under the induction of specific chemotherapy agents, which involves the phosphorylation of eukaryotic translation initiation factor eIF $2 \alpha$ by PKR-like ER kinase. This is followed by caspase-8-mediated proteolysis of the ER-sessile protein BAP31, activation of proapoptotic proteins BAX and BAK, anterograde transport of CRT from the ER to the Golgi apparatus, and the exocytosis of CRT-containing vesicles, eventually resulting in SNARE-dependent translocation of CRT to the plasma membrane surface. ${ }^{24,33}$ However, when the ER undergoes a stress response under the action of other ICD inducers, such as the reactive oxygen species (ROS) induced by PDT, the transport of CRT to the cell surface may not necessarily be completely achieved through the above pathways. ${ }^{24,34}$ These findings suggest that the exposure of CRT at the cell surface may be the net result of heterogeneous signaling pathways that are elicited in a stimulus-dependent manner.

In the context of the immune system, the prominent function of CRT is as an "eat-me" signal. Exposed CRT has been shown to bind to CD91, a transmembrane receptor. Thus, CRT-exposing cells can be recognized and engulfed by CD91-positive cells (mostly macrophages and dendritic cells (DCs), which are both antigenpresenting cells (APCs)). ${ }^{32}$ Then, the tumor antigens are processed and presented by APCs and prime a cognate immune response (Figure 2). Therefore, CRT exposure during ICD has great potential for eliciting antitumor immune responses.

\section{HMGBI Release}

HMGB1 is a highly conserved nuclear protein widely distributed in mammalian cells, which can be actively secreted by cells of the innate immune system in response to pathogenic products and released by injured cells as they succumb to mechanical damage and necrosis. ${ }^{35}$ Many classical inducers of apoptosis, including most antineoplastic agents, can promote HMGB1 release. ${ }^{35}$ Most intracellular HMGB1 is normally bound to chromatin. In response to stress, HMGB1 will be released from cells owing to a loss of plasma membrane integrity. ${ }^{36}$

The released HMGB1 is considered to be a potent proinflammatory stimulus. ${ }^{35-37}$ It triggers proinflammatory responses by binding to the receptors on the surface of 


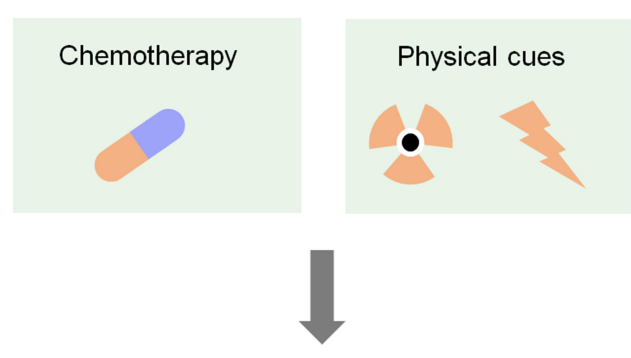

Immunogenic cell death

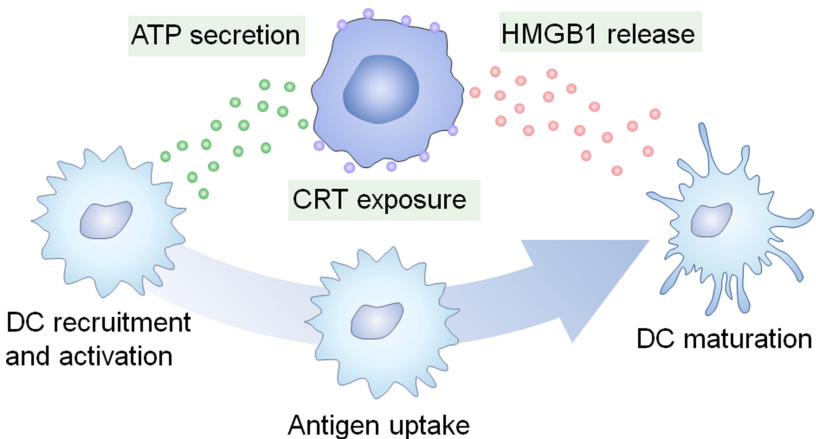

Figure 2 Certain chemotherapies and physical cues can induce tumor ICD. The dying tumor cells expose CRT, secrete ATP, and release HMGBI, all of which favor the recruitment, activation, and maturation of DCs.

immune cells. For instance, HMGB1 released from anthracycline-treated cancer cells can bind to TLR4, thereby activating the release of proinflammatory cytokines by monocytes or macrophages. ${ }^{38}$ Moreover, HMGB1 can promote the recruitment of inflammatory cells to inflammatory lesions by forming a complex with CXCL12 and signaling via CXCR $4 .{ }^{39}$ Importantly, productive immune responses against cancer cells require TLR4 as well as its adaptor MYD88. ${ }^{38,40}$ Treatment with HMGB1 augments the expression of pro-IL-1 $\beta$ in TLR4-expressing DCs, while the lysosomal degradation of engulfed tumor antigens is avoided, which is a major prerequisite for efficient cross-presentation. ${ }^{38}$ Therefore, the engagement of TLR4 by HMGB1 is critical to the optimal presentation of dead cell antigens by DCs.

\section{ATP Release}

ATP is the most abundant intracellular metabolite and plays an important part in purinergic neurotransmission. ${ }^{41}$ Besides, it can be released from cells under physical or chemical stresses, such as those caused by cytotoxic agents, hypoxia, mechanical distortion, or plasma membrane damage. It is possible that stress-induced ATP release occurs through the following mechanisms: active exocytosis of ATP-containing vesicles as well as secretion of cytoplasmic ATP via gap junction hemichannels (which are built up by connexins); pannexin channels; transporters of the ATP-binding cassette family; the cystic fibrosis transmembrane conductance regulator; and even P2X7 receptors, which are abundantly expressed by immune cells including macrophages and DCs. ${ }^{42}$

Although not sufficient, autophagy is required for the optimal release of ATP from dying tumor cells treated by chemotherapy. Autophagy-deficient tumor cells exhibit reduced ATP release in vivo, and local injections of ectonucleotidase inhibitors suffice to increase extracellular ATP levels in autophagy-deficient tumors. ${ }^{43}$ However, the exact molecular mechanisms through which autophagy contributes to ATP secretion remain elusive. ${ }^{44}$

Furthermore, ectonucleotidases including CD39 (which converts ATP into ADP and AMP) and CD73 (which converts AMP into the immunosuppressive metabolite adenosine) are important factors that reduce the local ATP concentration of tumors. ${ }^{45,46}$

ATP released from dying cells is one of the most prominent chemotactic "find-me" signals for recruitment of macrophages and DCs, probably owing to its binding to P2Y2 receptors (which are widely expressed on cells of the myeloid lineage). ${ }^{47,48}$ In response to specific chemotherapy agents, ATP released by tumor cells is positively correlated with the recruitment of monocytes, macrophages and DCs, eliciting antitumor immune responses and promoting optimal chemotherapy responses. ${ }^{43}$ Beyond its role as a chemoattractant, extracellular ATP may affect the function of immune effectors and has been shown to stimulate the maturation of murine bone marrow-derived DCs. ${ }^{49}$

\section{ICD Inducers Based on Nanoparticle Drug Delivery System} Although cancer cells undergo ICD in response to chemotherapy, radiation, or other physical cues, and the immune response is further activated, the applications of traditional ICD inducers are limited by challenges relevant to safety and efficacy. To address these problems, nanoparticles are used to protect the therapeutic agent from rapid clearance and enhance specific drug delivery to the tumor site, thereby improving the curative effect and reducing adverse events. In general, nanoparticles are divided into categories based on their physical and chemical properties, such as material type, size, shape, charge, and surface chemical modifications. ${ }^{50}$ For instance, various synthetic polymers, biomimetic materials, and inorganic 
materials, have been used for the construction of nanoparticles. In particular, nanoparticles composed of synthetic polymers can be molded to create the desired structure, but their poor penetration ability and uptake by immune cells limits their applications. ${ }^{51}$ Nanoparticles composed of biomimetic materials, such as coating with cell membrane, show improved biocompatibility and targeting ability with reduced immunologic rejection. However, the complexity of their preparation presents difficulties for their clinical applications. ${ }^{52}$ Inorganic nanoparticles have several advantages, including being relatively stable and easy to synthesize; they also exhibit some special properties, such as photothermal conversion capability, photodynamic properties, and superparamagnetic properties. Nevertheless, common inorganic nanoparticles are difficult to degrade in vivo and are associated with potential safety concerns. In addition to the application of nanoparticles in drug delivery, controllable drug release at the lesion is important. Therefore, smart nanoparticles, which can be triggered to release a drug under specific conditions (light, hyperthermia, $\mathrm{pH}$, enzymes, or redox), ${ }^{53}$ have also attracted much attention. In the field of cancer combination therapy, each type of nanoparticle mentioned above offers unique advantages and applications, especially for use in drug co-delivery systems, which can simultaneously deliver more than one drug to a tumor site, thereby facilitating cancer combination therapy. In this review, we summarize some nanoparticle-based ICD inducers that can increase the tumor accumulation of drugs and increase tumor ICD.

\section{ICD Elicited by Nanoparticle-Based Chemotherapy}

Chemotherapy is one of the most widely used treatment modalities for most advanced cancers in clinical settings. Several chemotherapy agents, including anthracyclines, cyclophosphamide, OXA, and microtubular inhibitors (such as taxanes and vinca-alkaloids), can successfully promote tumor ICD and further stimulate anticancer immune responses. ${ }^{16,44,54}$ Inspired by this property, nanoparticle-based chemotherapy has been developed to amplify the ICD induction of these drugs, while eliminating their systemic toxicity to normal tissues. Zhao et $\mathrm{al}^{17}$ applied monomethoxy-poly(ethylene glycol)-poly(D, L-lactide-co-glycolide) (mPEG-PLGA) polymeric nanoparticles as model nanocarriers to encapsulate OXA (an ICD inducer) or gemcitabine (a non-ICD inducer), both of which are used clinically as first-line chemotherapy regimens for pancreatic cancer. ${ }^{55}$ Interestingly, tumor cells treated with nanoparticle-encapsulated OXA released more DAMPs and induced stronger immune responses of DCs and T lymphocytes than those treated with free OXA in vitro. Furthermore, the therapeutic efficacy in immunocompetent mice showed the same improvement. Although nanoparticle encapsulation did not endow a non-ICD inducer with ICD-mediated antitumor capacity, treatment with a nanoparticle-encapsulated ICD inducer led to significantly enhanced ICD and consequently improved antitumor effects compared with the free ICD inducer, corroborating the general trend of ICD enhancement with nanoparticle delivery. When nanoparticles are modified with tumor-targeting motifs, their tumor accumulation can be further facilitated. Qi et al $^{56}$ developed an innovative chemo-immunostrategy based on targeted delivery of mitoxantrone (MIT) and celastrol (CEL), two potent medicines screened and selected as having the best anticancer and antifibrosis potential. AEAA-polymer-disulfide-bond nanoparticles synthesized by Michael addition polymerization were applied for drug delivery; AEAA is a molecule with tumor targeting ability. Importantly, CEL worked in synergy with MIT to induce ICD and recover tumor antigen recognition by immune cells, thereby eliciting overall antitumor immunity. Furthermore, the strong synergy benefitted the host in terms of reduced drug exposure and side effects. The nanoparticle-mediated chemo-immunotherapy successfully remodeled the fibrotic and immunosuppressive tumor microenvironment, arrested cancer progression, and further inhibited tumor metastasis to major organs. The tumor targeting of nanoparticles can also be promoted by external factors, such as a magnetic field. Alev et $\mathrm{al}^{5}$ developed an iron oxide nanoparticle-based system for the magnetically targeted delivery of MIT to tumors, which could induce ICD and concomitant maturation of DCs. Thus, the iron oxide nanoparticle-based system is a potential platform to deliver other ICD inducers to tumor regions, while avoiding toxic effects. Chen et al ${ }^{13}$ designed tumor-targeting core-shell magnetic nanoparticles (ETP-PtFeNP) to reinforce ICD induction of an OXAloaded prodrug, leading to enhanced ICD-associated immunogenicity and specific antitumor immune responses to kill tumor cells synergistically (Figure 3).

In addition to traditional chemotherapy agents, some cationic anticancer peptides can also induce ICD and promote antitumor immunotherapy. Qi et $\mathrm{al}^{57}$ used $\mathrm{pH}$ and thermal dual-sensitive nanoparticles based on poly 


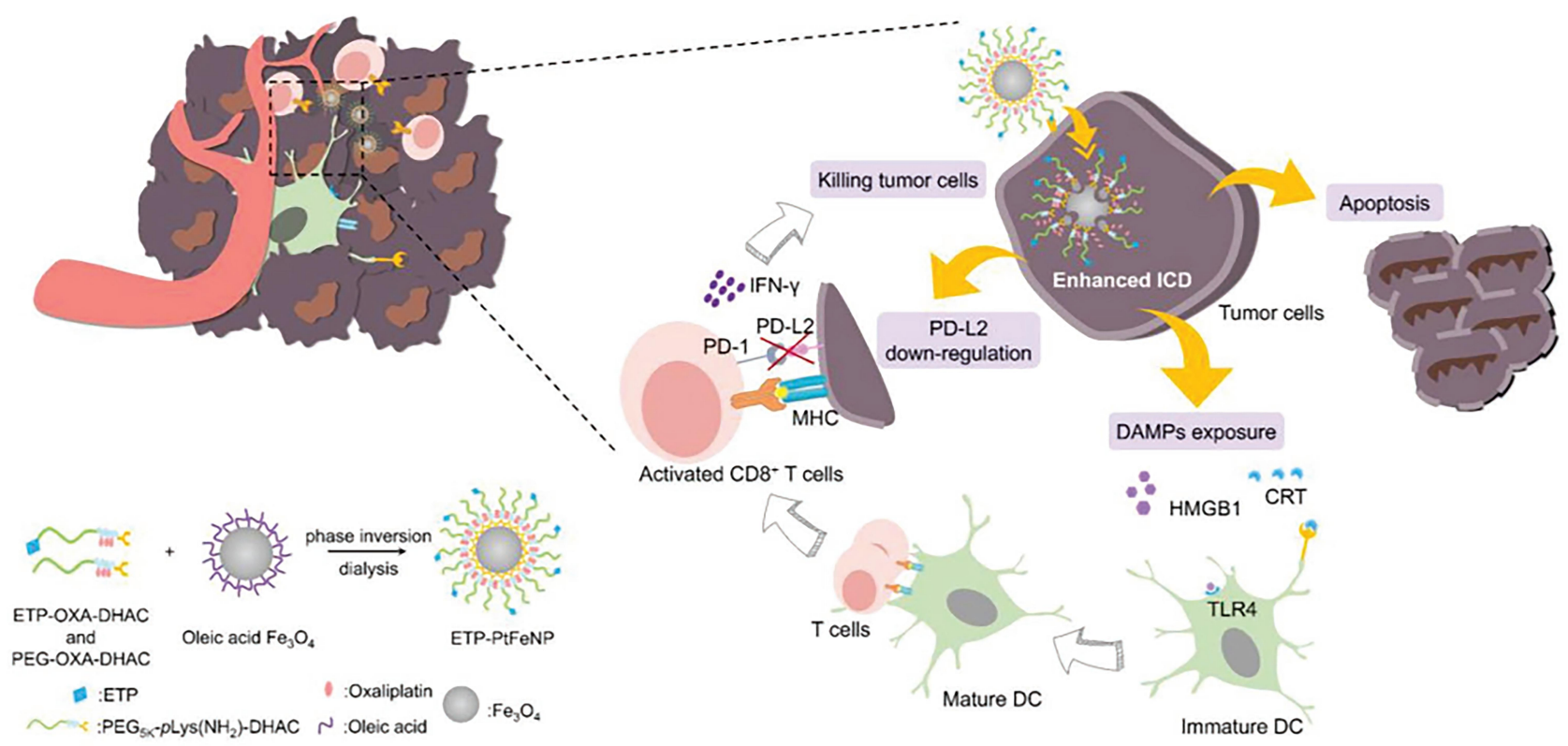

Figure 3 The tumor-targeting core-shell magnetic nanoparticles (ETP-PtFeNP) could kill tumor cells, accompanied by enhanced ICD, the DAMPs exposure could promote the maturation of DCs and further facilitate the specific antitumor immune responses. Adapted from Chen Q, Liu L, Lu Y, et al. Tumor microenvironment-triggered aggregated magnetic nanoparticles for reinforced image-guidedimmunogenic chemotherapy. Adv Sci (Weinh). 2019;6:1802I34. (C) 2019 The Authors. Published by WILEYVCH Verlag GmbH \& Co. KGaA, Weinheim. Creative Commons CC BY license. ${ }^{13}$

(acrylamide-co-acrylonitrile)-PEG and hyaluronic acid to deliver bovine lactoferricin (LfcinB, one of the most widely studied cationic anticancer peptides) to tumor sites. The released LfcinB could induce tumor apoptosis with the release of DAMPs and further elicited a tumor-specific immune response. Consequently, tumor growth was inhibited significantly compared with treatment with free LfcinB.

Collectively, nanoparticle-based chemotherapy not only improves biodistribution and biocompatibility of drugs but also provides a highly effective approach for ICD induction, achieving activation of antitumor immune responses and outstanding therapeutic efficacy. Nanoparticle delivery systems are thus of great significance in the field of tumor chemo-immunotherapy.

\section{ICD Elicited by Nanoparticle-Based Chemotherapy Combined with Immune Checkpoint Inhibitors}

The tumor immunosuppressive microenvironment is an important factor limiting the effect of tumor treatment, and immune checkpoint inhibitors are expected to be an effective means of alleviating this phenomenon. Therefore, the combination of targeted ICD inducers and immune checkpoint inhibitors is a promising strategy to improve antitumor effects.

Kuai et $\mathrm{al}^{58}$ showed that high-density-lipoproteinmimicking nanodiscs loaded with doxorubicin (DOX), a widely used chemotherapy agent, could trigger ICD of cancer cells and exert antitumor effects without any overt off-target side effects. Besides, the DOX could potentiate immune checkpoint blockade in murine tumor models. Thereafter, the combination of nanodiscs and anti-PD-1 therapy induced complete regression of established CT26 and MC38 colon carcinoma tumors in $80-88 \%$ of animals and protected survivors against tumor recurrence. This work provides a generalizable framework for using nanoparticle-based chemotherapy to initiate antitumor immunity and sensitize tumors to immune checkpoint blockade. $\mathrm{Lu}$ et $\mathrm{al}^{59}$ constructed a liposome self-assembled from phospholipid-conjugated prodrug indoximod (IND), which inhibits the IDO-1 pathway, followed by remote loading of ICD-inducing chemotherapy drug DOX. They used this liposomal drug delivery system to simultaneously trigger ICD and relieve the immunosuppression caused by regional overexpression of IDO-1 at the tumor site. Not only did the DOX/IND-liposome provide a synergistic antitumor response superior to that achieved with a DOX-only liposome, it also demonstrated that the carrier could be effectively combined with PD-1-blocking antibodies to eradicate lung metastases. Moreover, nanoparticles sensitivities play an important part in controlled drug release. Feng et $\mathrm{al}^{60}$ demonstrated a tumor acidity and reduction microenvironment dual-activatable binary 
cooperative prodrug nanoparticle $(\mathrm{BCPN})$ to deliver OXA and NLG919 (an IDO-1 inhibitor) for chemoimmunotherapy. The exposed OXA promoted tumor infiltration of cytotoxic $\mathrm{T}$ lymphocytes by triggering ICD of cancer cells, while the NLG919 downregulated IDO1-mediated immunosuppression and suppressed regulatory $\mathrm{T}$ cells. Given its merits of high drug loading efficacy, tumor-specific accumulation and deep penetration, and burst drug release inside tumor cells, as well as its robust formulation procedure, this prodrug nanoparticle has potent potential for improving immunotherapy (Figure 4). Recent research on the cancer combination therapy of nanoparticle-based chemical ICD inducers and immune checkpoint inhibitors is summarized in Table 1.

In conclusion, nanoparticle-based chemotherapy can effectively improve tumor immunogenicity, while immune checkpoint inhibitors can alleviate tumor immunosuppression; therefore, the combination strategy will achieve remarkable antitumor effects.

\section{ICD Activated by Nanoparticle-Based Physical Cues}

In addition to chemotherapy, some physical stimuli can cause ICD in tumor sites; these include radiotherapy such as X-rays, $\gamma$-rays, protons, or electron beams; and phototherapy including PTT and PDT. In radiotherapy, the ionizing radiation can directly break the DNA structure or generate ROS that further damage the DNA, resulting the activation of tumor ICD and an immune response. ${ }^{66,67}$ Regarding phototherapy, the new emerging cancer treatments usually employ phototherapeutic agents to selectively kill tumor cells under certain light irradiation. ${ }^{68,69}$ PTT usually leverages optical absorbing agents to generate hyperthermia under light irradiation to "burn" cancer cells. ${ }^{70}$ PDT relies on photosensitizers that convert the surrounding oxygen molecules to ROS or cytotoxic singlet oxygen $\left({ }^{1} \mathrm{O}_{2}\right)$ to kill cancer cells under the appropriate light irradiation. ${ }^{71}$ Both PTT and PDT can directly kill tumor cells, accompanied by release of DAMPs, which can further induce a strong antitumor immune response. Importantly, when nanoparticle delivery systems are combined with these physical therapies, the area, time, and efficacy of treatment can be precisely controlled by changing the site, duration, and power of irradiation. As a result, it is easy to adjust the treatment strategy to induce effective tumor ICD in a timely manner according to clinical needs. $^{72,73}$

There have been many studies of tumor ICD activated by physical cues based on nanoparticle drug delivery
A

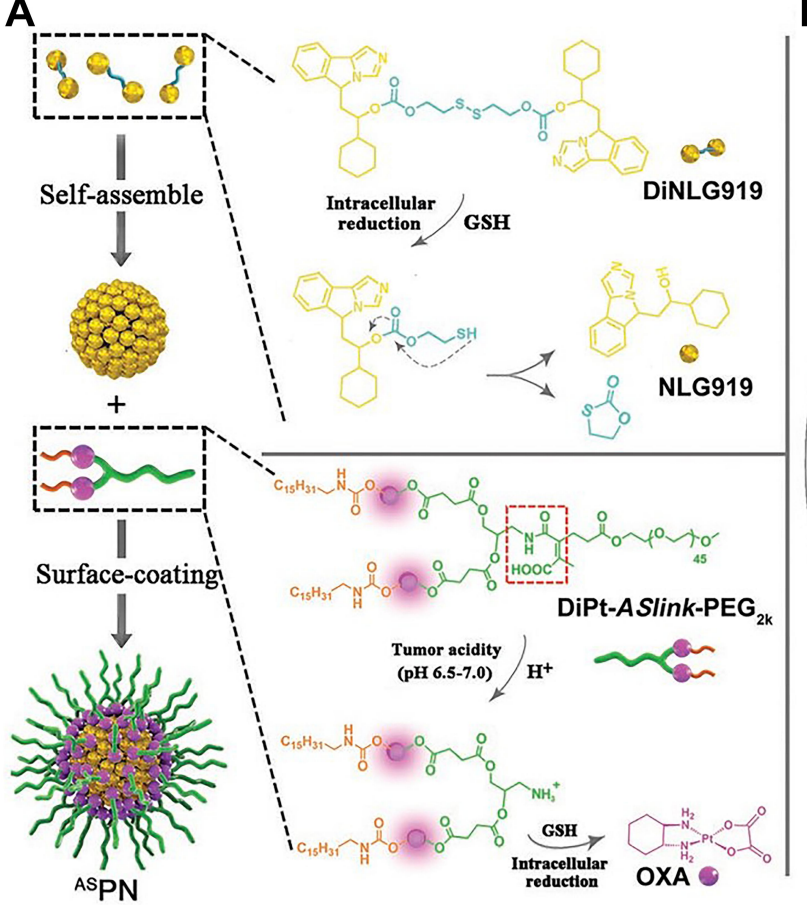

B

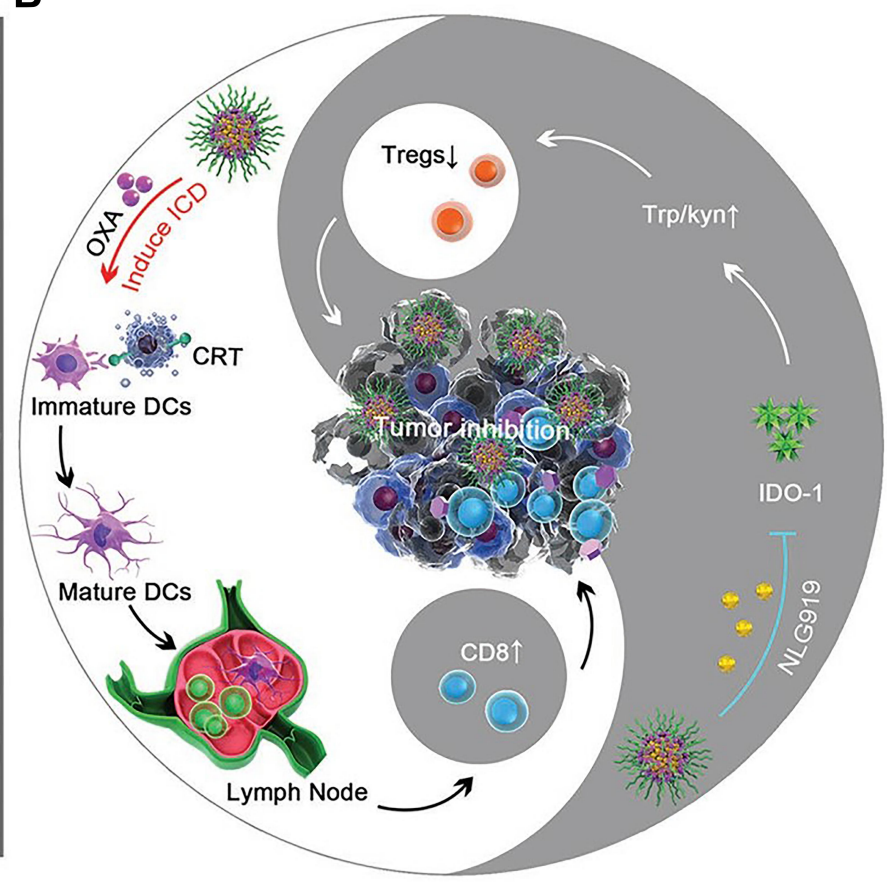

Figure 4 Schematic illustration of the BCPN for improved immunotherapy by cooperatively modulating the immune tumor microenvironment. (A) Self-assembly procedure of BCPN nanoparticles, and chemical structure of DiNLG919 and prodrugs. (B) Schematic illustration of BCPN to elicit antitumor immunity and suppress regulatory T cells for improved immunotherapy. Adapted with permission from Feng B, Zhou F, Hou B, et al. Binary cooperative prodrug nanoparticlesimprove immunotherapy by synergistically modulatingimmune tumor microenvironment. Adv Mater. 2018;30:1803001. Copyright @ 2018 John Wiley and Sons. ${ }^{60}$ 


\begin{tabular}{|c|c|c|c|c|c|}
\hline 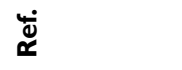 & 总 & 宫 & 实 & $\overline{\bar{b}}$ & శ్ర \\
\hline 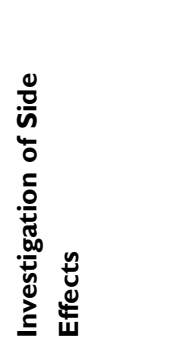 & 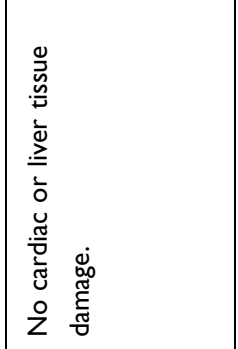 & 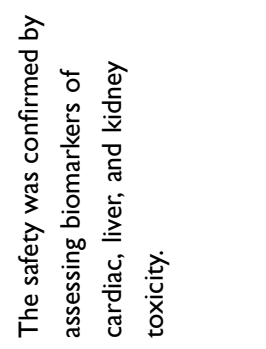 & 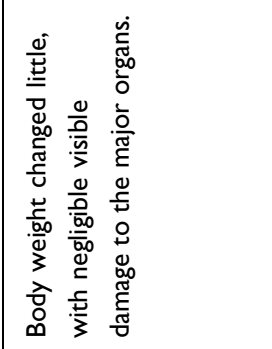 & 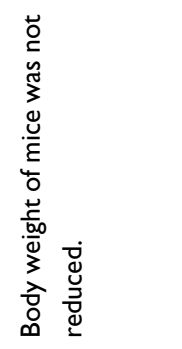 & 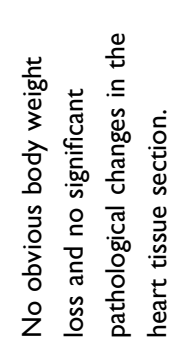 \\
\hline 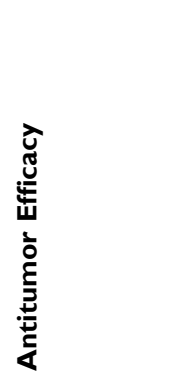 & 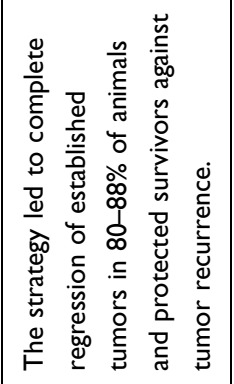 & 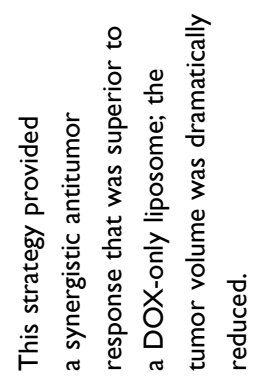 & 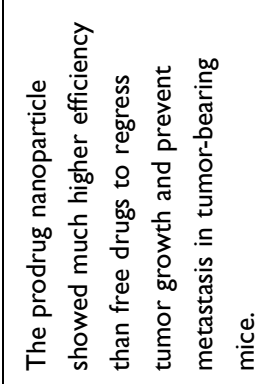 & 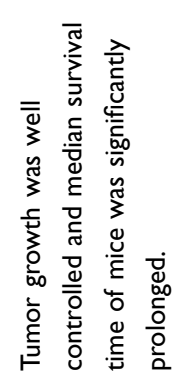 & 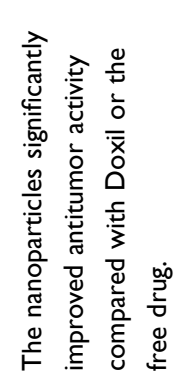 \\
\hline 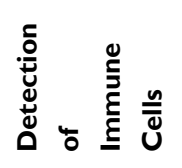 & $\begin{array}{l}\overline{\bar{\Xi}} \\
\leftarrow \\
\vdash \\
+\infty \\
0 \\
0\end{array}$ & 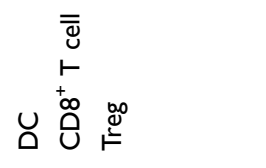 & 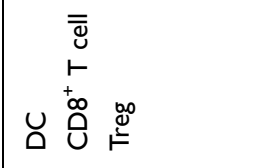 & 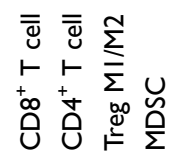 & 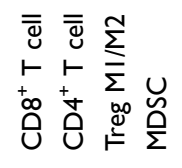 \\
\hline 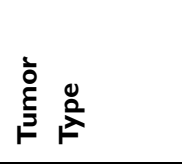 & 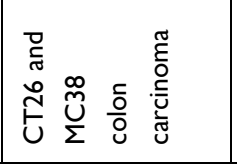 & 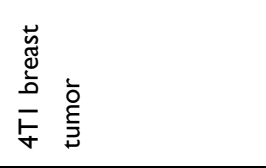 & 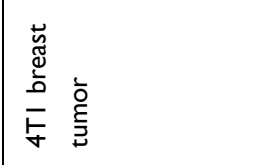 & 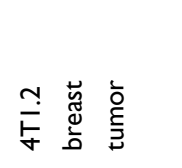 & 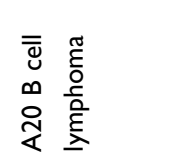 \\
\hline 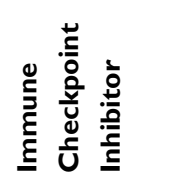 & 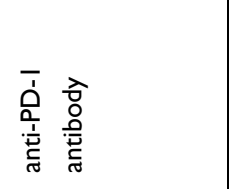 & 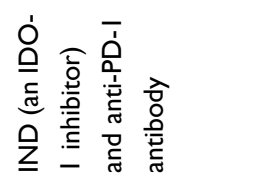 & 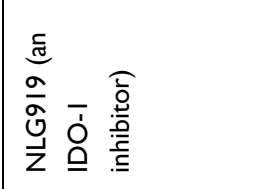 & 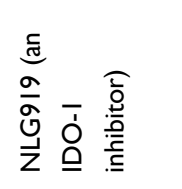 & 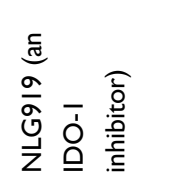 \\
\hline 品 & 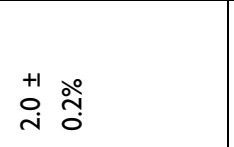 & 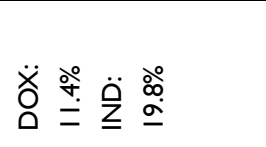 & 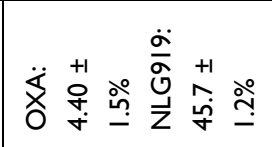 & 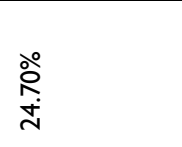 & $\begin{array}{l}\text { 今ें } \\
\text { 요 }\end{array}$ \\
\hline 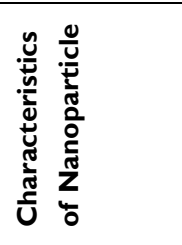 & 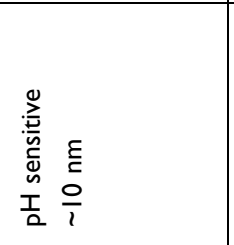 & 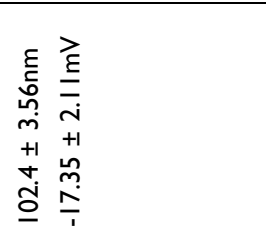 & 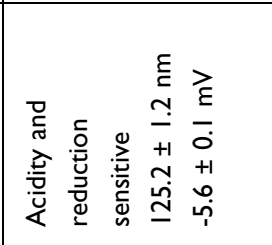 & 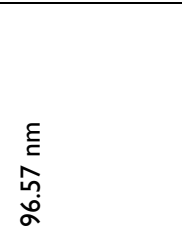 & 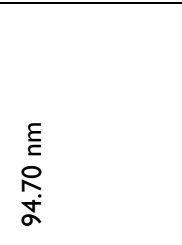 \\
\hline 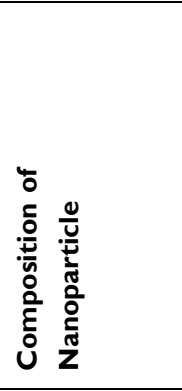 & 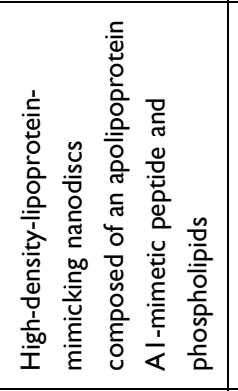 & 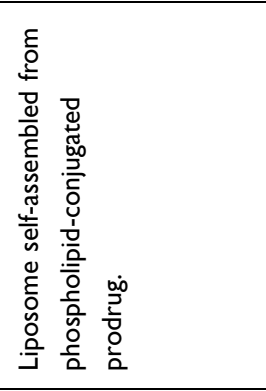 & 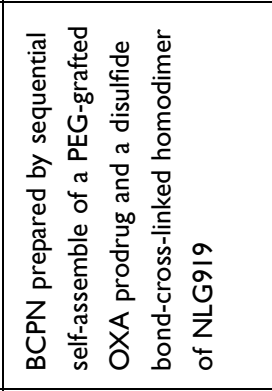 & 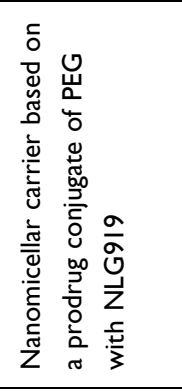 & 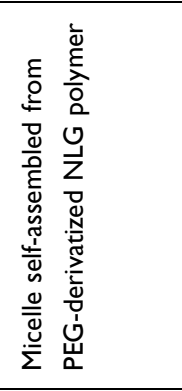 \\
\hline 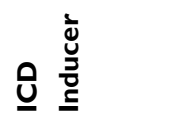 & ํ. & ○̊ & 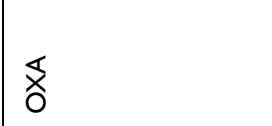 & 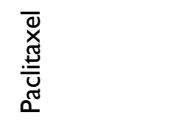 & ○̊ \\
\hline
\end{tabular}




\begin{tabular}{|c|c|c|}
\hline 苞 & 悉 & 局 \\
\hline 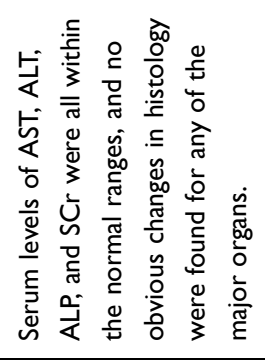 & - & - \\
\hline 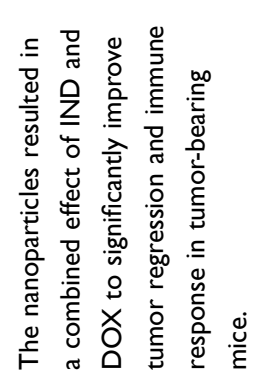 & 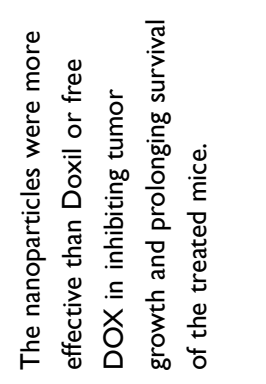 & 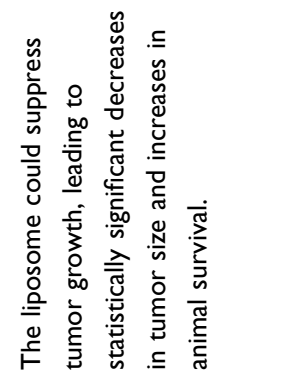 \\
\hline 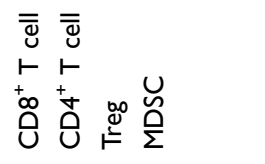 & 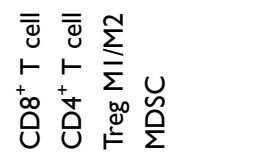 & 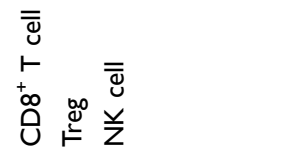 \\
\hline 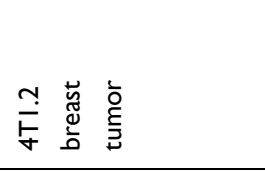 & 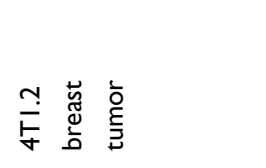 & 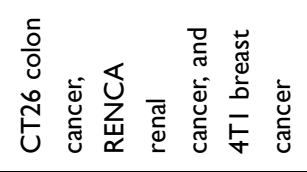 \\
\hline 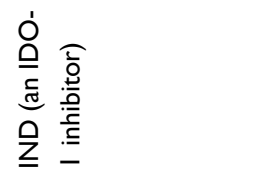 & 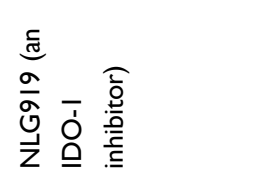 & 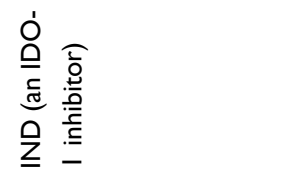 \\
\hline 高 & 官 & @̈ \\
\hline 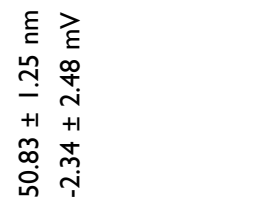 & 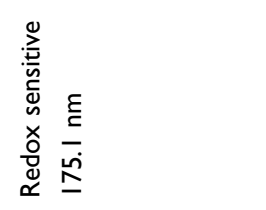 & 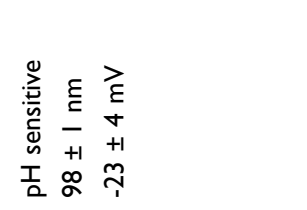 \\
\hline 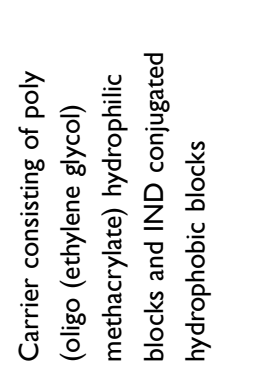 & 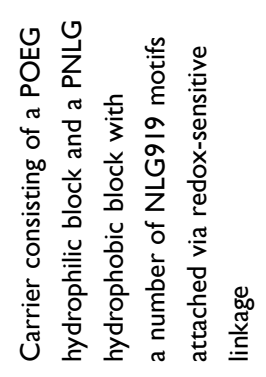 & 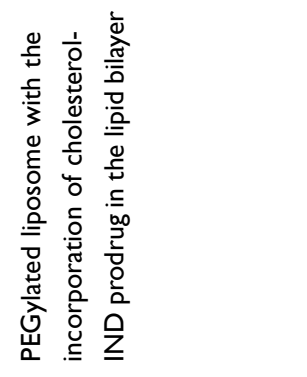 \\
\hline ox & ơ & $\stackrel{上}{\Sigma}$ \\
\hline
\end{tabular}


systems. Vanpouille-Box et $\mathrm{al}^{74}$ reported a lipid nanocapsule loaded with rhenium-188 (LNC ${ }^{188} \mathrm{Re}-\mathrm{SSS}$ ) to achieve local radiotherapy for tumors with reduced systemic toxicity. The danger signals released by irradiated tumor cells could recruit and activate immune cells, further enhancing the effect of this treatment strategy. Zhang et $\mathrm{al}^{75}$ constructed a magnetic delivery system for effective cancer treatment via PTT. This delivery system was composed of $\mathrm{Fe}_{3} \mathrm{O}_{4}$ magnetic nanoparticles as the core to load indocyanine green (ICG), and polyethylene glycol polyphenols as the coating layer to load R837, a Toll-like-receptor-7 agonist that serves as an immune adjuvant. When the nanoparticles were targeted to the tumor site and irradiated by near-infrared light, tumor-associated antigens were released under the PTT and the antitumor immunological response was further promoted by R837. There are also some instances of PDT; Chen et $\mathrm{al}^{76}$ presented a protein hybridization approach to develop a bioinspired hybrid protein oxygen nanocarrier with chlorin e6 (Ce6, a photosensitizer) loaded via intermolecular disulfide conjugation for oxygen-augmented immunogenic PDT. The nanocarrier could co-deliver the photosensitizer and oxygen to tumor sites, which markedly relieved tumor hypoxia. The PDT efficiency was enhanced and ICD of cancer cells was induced. Notably, immunogenic PDT mediated by the fabricated nanocarrier could destroy primary tumors and effectively suppress distant tumors and lung metastasis in a metastatic triple-negative breast cancer model by evoking systemic antitumor immunity. This study provides a paradigm of oxygen-augmented immunogenic PDT for metastatic cancer treatment. Besides, Wang et $\mathrm{al}^{77}$ designed a ultrasmall nanoagent fabricated from polyethylene-glycol-modified $\mathrm{Cu}_{2-\mathrm{x}} \mathrm{Se}$ nanoparticles, $\beta$ cyclodextrin, and Ce6. The resultant nanoplatform could be passively accumulated into the tumor and exhibited dramatic antitumor efficacy owing to an excellent PDT effect under near-infrared irradiation. Subsequently, the vast amounts of ROS generated not only killed primary tumor cells but also elicited ICD to release DAMPs and induced polarization of proinflammatory M1macrophages. Thereby, robust antitumor immune responses against the metastasis of breast cancer were evoked. In addition, the combination of PTT and PDT has been investigated. Based on the fact that ICD can be evoked through ROS produced via ER stress, $\mathrm{Li}$ et $\mathrm{al}^{78}$ generated a double ER-targeting strategy to realize PDT/ PTT/immunotherapy. ER-targeting pardaxin (FAL)peptide-modified, ICG-conjugated-hollow gold nanospheres (FAL-ICG-HAuNS), together with an oxygen-delivering hemoglobin liposome (FAL-Hb lipo), were designed to induce robust ER stress and ICD. This strategy successfully promoted the exposure of CRT and activated a series of immunological responses, resulting in enhanced antitumor efficacy (Figure 5).

Moreover, core-shell-structured nanoparticles have been applied widely. He et $\mathrm{al}^{79}$ developed a core-shell gold nanocage coated with manganese dioxide $\left(\mathrm{MnO}_{2}\right)$ and hyaluronic acid for targeted delivery to colorectal tumors and oxygenation-boosted immunogenic phototherapy in situ. In this nanoplatform, the gold nanocage core was employed for near-infrared light (NIR)-triggered PDT, while the $\mathrm{MnO}_{2}$ shell was degraded in the tumor microenvironment containing acidic $\mathrm{H}_{2} \mathrm{O}_{2}$, and generated sufficient oxygen to ameliorate tumor hypoxia and to increase ROS production and PDT efficacy. This strategy enhanced tumor ICD and facilitated DC maturation, which effectively inhibited the growth and recurrence of colorectal tumors. Cell-membrane-coated nanoparticles have also attracted much attention. Yu et $\mathrm{al}^{80}$ developed a myeloidderived suppressor cell (MDSC) membrane-coated iron oxide magnetic nanoparticle (MNP@MDSC) for active tumor targeting and PTT-induced tumor killing. MNP@MDSC could act as a PTT agent to achieve an enhanced antitumor response by inducing ICD, reprogramming tumor-infiltrating macrophages, and reducing the tumor's metabolic activity, making it a promising agent for cancer therapy.

Tumor ICD induced by nanoparticle-based physical means can effectively stimulate an antitumor immune response, and has great potential in the field of tumor treatment. Nanoparticle drug delivery systems contribute significantly to improvements in tumor targeting and the antitumor efficacy of physical cues.

\section{ICD Activated by Nanoparticle-Based Physical Cues Combined with Immune Checkpoint Inhibitors}

Similarly, the antitumor efficacy of these nanoparticlebased physical cues is limited by tumor immunosuppression, even though the tumor ICD has been activated. Therefore, the combined application of immune checkpoint inhibitors will be an effective way to solve this problem. ${ }^{81}$

Regarding the application of radiotherapy, Choi et al ${ }^{82}$ synthesized a splintery snowflake-like $\mathrm{Au}$ nanocarrier 


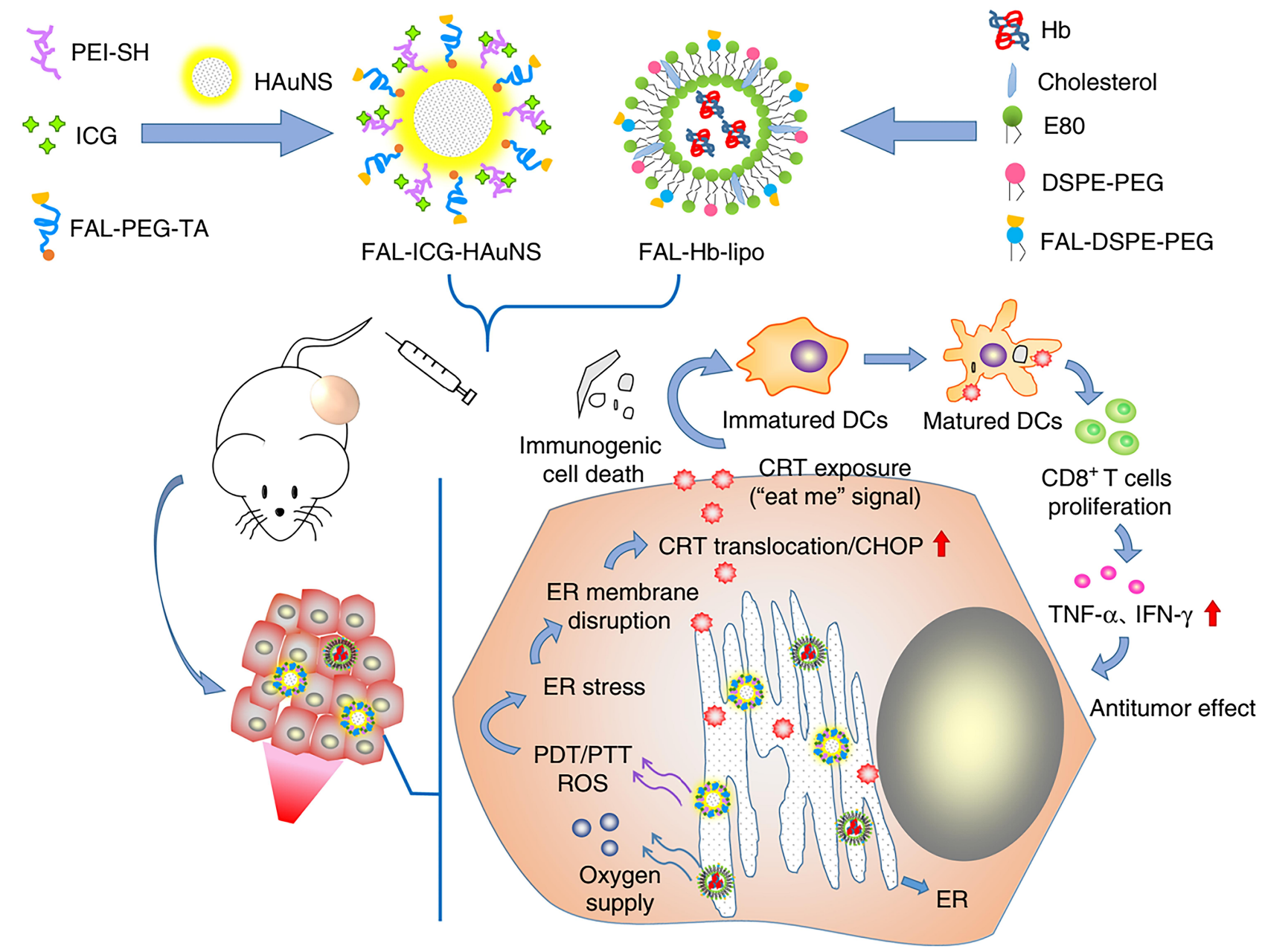

Figure 5 The antitumor mechanism of FAL-ICG-HAuNS plus FAL-Hb-lipo. Schematic illustration of enhanced immunogenic cancer cell death and anticancer effect induced by endoplasmic reticulum-targeting photothermal/photodynamic therapy. Adapted from.$^{78}$

(S-AuNC) that could increase the selectivity of radiation and showed radiation-responsive structural deformation, resulting in the triggered release of the cargo, $\alpha$ PD-L1. The combination of radiotherapy and $\alpha \mathrm{PD}-\mathrm{L} 1-$ loaded S-AuNC significantly enhanced the ICD for tumor microenvironment conversion, resulting in a synergistic anticancer adaptive immune response with minimized systemic immune-related adverse effects. Yu et al $^{83}$ prepared electric pulse responsive iron-oxide-nanocube clusters (IONCs) loaded with IDO inhibitors (IDOi) to enhance cell killing by irreversible electroporation (IRE) and modulate the tumor immunosuppressive microenvironment. The IDOi-loaded IONCs showed highly responsive movement upon application of IRE electric pulses that induced local magnetic fields, further enhancing tumor-killing efficiency accompanied by ICD, and the released IDOi could overturn the tumor immunosuppressive phenomenon. Yang et $\mathrm{al}^{84}$ designed $\mathrm{pH}$-responsive nanovesicles to encapsulate a photosensitizer (HPPH) and an IDO inhibitor (IND) via hydrophobic interactions. The obtained nanoparticles (pRNVs/HPPH/IND) could directly kill cancer cells by PDT under laser irradiation and induce ICD. On the other hand, IND could restore the mTOR pathway, with phosphorylation of P-S6K for tumor microenvironment modulation, which eventually stimulated $\mathrm{CD}^{+}$ $\mathrm{T}$ cells. This strategy provides huge potential for design of nanomaterials for cancer immunotherapy. Liu et $\mathrm{al}^{85}$ reported a serum-albumin (SA)-coated boehmite organicinorganic scaffold loaded with $\mathrm{Ce} 6$ and a honeybee venom melittin (MLT) peptide, denoted Ce6/MLT@SAB. With the assistance of MLT, Ce6/MLT@SAB could more easily accumulate in tumor cells, permitting greater intercellular ROS production by PDT and further enhancing the ICD effect. It is worth noting that when the Ce6/MLT@SAB was used in combination with a PD-1 inhibitor, the numbers of $\mathrm{CD}^{+}$and $\mathrm{CD}^{+} \mathrm{T}$ cells in tumors further 
increased, whereas numbers of MDSCs were reduced, indicating that the addition of anti-PD-1 immunotherapy could strengthen the antitumor ability of Ce6/MLT@SAB phototreatment to achieve the desired therapeutic effect (Figure 6). Recent research on combination cancer therapies using nanoparticle-based physical ICD inducers and immune checkpoint inhibitors is summarized in Table 2.

In conclusion, these studies present unique nanomedicine approaches representing a next-generation tumor therapy strategy that can activate synergistic whole-body therapeutic responses and overturn tumor immunosuppression when combined with immune checkpoint inhibitors, holding great promise for clinical translation.

\section{Multipronged Approaches}

In order to evoke a significant ICD at the tumor site, immunogenic chemotherapy has also been combined with other physical cues, such as PTT and PDT, to achieve a synergistic effect. Importantly, when the synergistic strategy of chemical and physical cues is combined with immune checkpoint inhibitors, a remarkable antitumor immune response is elicited, further improving the antitumor efficacy.

The combination of chemotherapy and PTT has a significant role in promoting tumor ICD. Wen et al ${ }^{89}$ proposed a new therapeutic strategy to effectively trigger ICD by combining chemotherapy and PTT. A nanosystem (Pd-DOX @TGMs nanoparticles) was fabricated by integrating DOX and photothermal reagent palladium nanoparticles (Pd NPs) into amphiphile triglycerol monostearates (TGMs). It was proved that codelivery of DOX and Pd NPs could effectively kill tumor cells, accompanied by the release of DAMPs to improve the immunogenicity of dead tumor cells. The

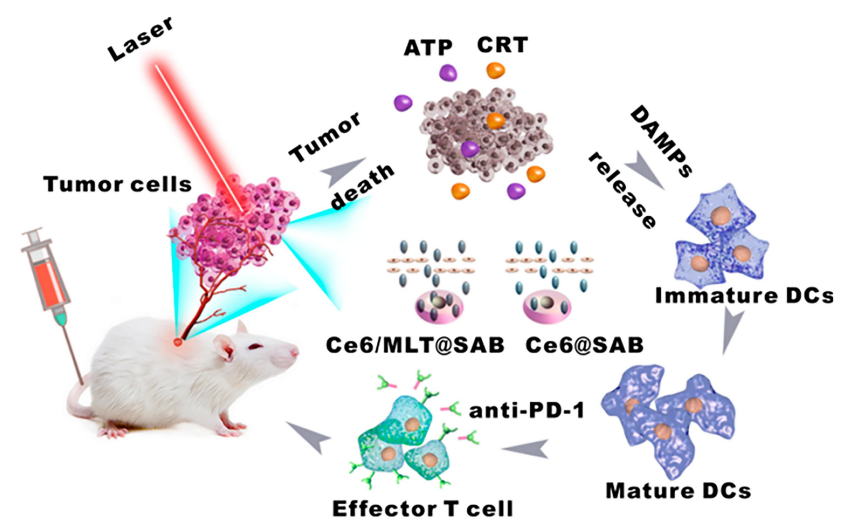

Figure 6 Depiction of an organic-inorganic hybrid nanocarrier coloaded with bee venom MLT and a photosensitizer for combinational photodynamic therapy and immunotherapy. Adapted with permission from . Copyright (c) 2019 American Chemical Society. ${ }^{85}$ effective ICD induction mediated by the nanosystem boosted the PD-L1 checkpoint blockade effect, which efficiently improved the infiltration of toxic T lymphocytes at the tumor site, resulting in excellent tumor treatment effects on both primary and abscopal tumors. Besides, Sun et $\mathrm{al}^{90}$ prepared a novel polypyrrole nanoparticle using the near-infrared dye IRDye800CW with a camptothecin (CPT)-conjugated hyaluronic acid (HA) shell (PPy@CPT-HA-IRDye800CW) for synergistic chemotherapy and PTT, which could induce tumor ICD and consequently evoke a systemic immune response. The antitumor therapeutic effects were synergistically enhanced when it was used in combination with anti-PD-L1 therapy, eliminating the primary tumor and preventing metastases and recurrences in tumor-bearing mice.

In addition, the combination of chemotherapy and PDT has also attracted attention. He et $\mathrm{al}^{91}$ demonstrated the synergistic ability of chemotherapy and PDT to elicit antitumor immunity. A nanoscale coordination polymer (NCP) core-shell nanoparticle was constructed, carrying OXA in the core and photosensitizer pyropheophorbide-lipid conjugate (pyrolipid) in the shell (NCP@pyrolipid). The synergy between OXA and the pyrolipid-induced PDT could induce tumor ICD and evoke an immune response. Importantly, NCP@pyrolipid treatment in combination with PD-L1 checkpoint blockade therapy not only led to the regression of primary tumors (treated locally with light irradiation) but also resulted in the regression of distant tumors in bilateral syngeneic tumor-bearing mice by generating a systemic tumor-specific T-cell response with infiltration of $\mathrm{CD}^{+}$ $\mathrm{T}$ cells and $\mathrm{CD}^{+} \mathrm{T}$ cells into distant tumors. Yang et $\mathrm{al}^{92}$ developed an intelligent PEG-modified, biodegradable hollow $\mathrm{MnO}_{2}\left(\mathrm{H}-\mathrm{MnO}_{2}\right.$-PEG) nanoplatform for co-loading a photodynamic agent $\mathrm{Ce} 6$ and a chemotherapy drug DOX. The obtained $\mathrm{H}-\mathrm{MnO}_{2}-\mathrm{PEG} / \mathrm{C} \& \mathrm{D}$ could be dissociated under reduced $\mathrm{pH}$ within tumor microenvironment to release loaded therapeutic drugs, while simultaneously decomposing tumor endogenous $\mathrm{H}_{2} \mathrm{O}_{2}$ to relieve tumor hypoxia. As a result, a remarkable in vivo synergistic therapeutic effect was achieved through combined chemo-photodynamic therapy, which simultaneously triggered a series of antitumor immune responses due to the ICD effect. The further combination of this platform with PD-L1 checkpoint blockade therapy would lead to inhibition of tumors at distant sites, as a potential treatment for tumor metastasis. Zhou et $\mathrm{al}^{93}$ reported a tumor-microenvironment-activatable prodrug vesicle, engineered by integrating an OXA prodrug and PEGylated photosensitizer ( $\mathrm{PPa}$ ) into a single nanoplatform. When the prodrug vesicle accumulated at the tumor site and 


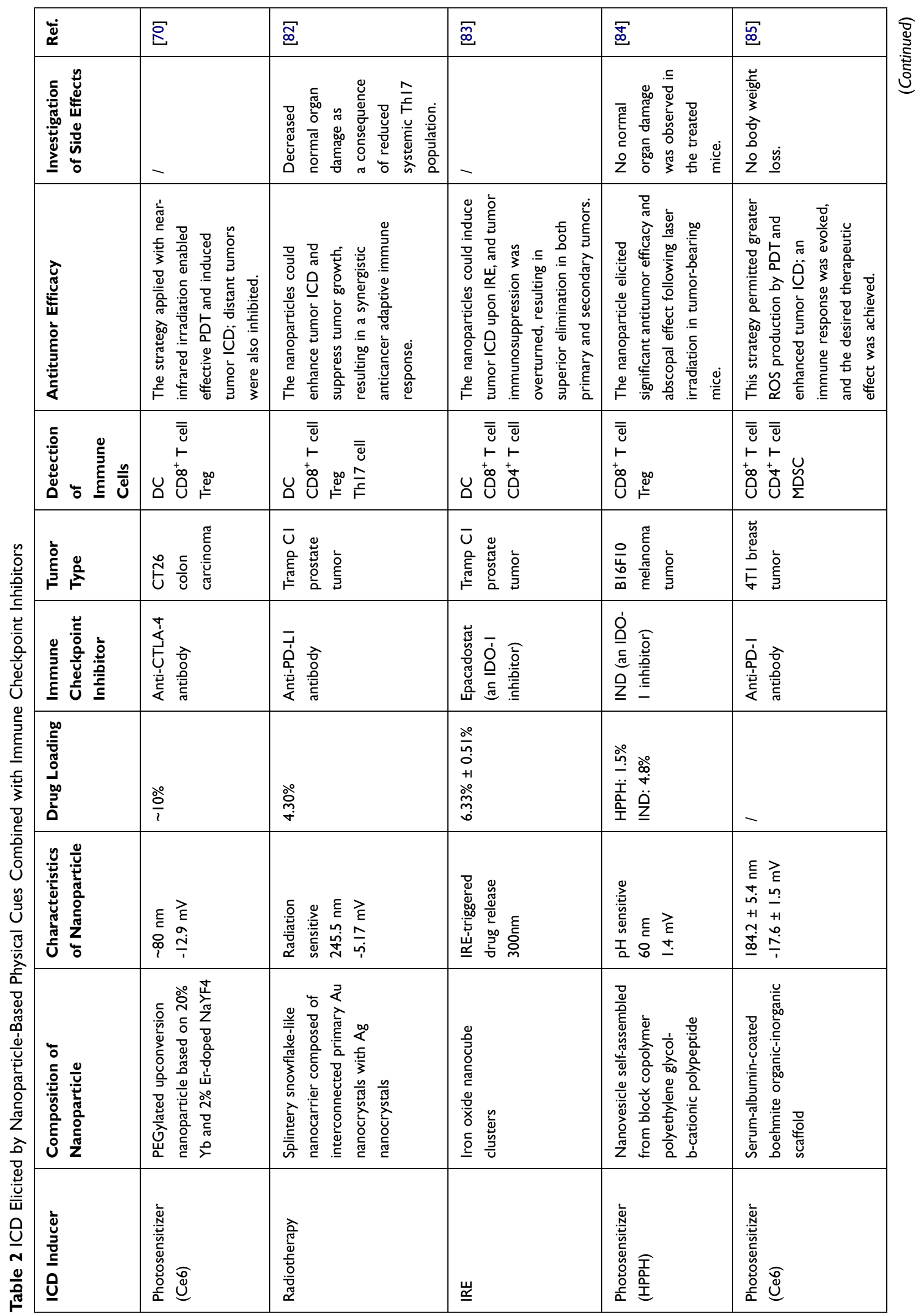




\begin{tabular}{|c|c|c|c|}
\hline 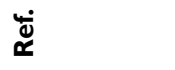 & 总 & $\underset{\infty}{\mathbb{\infty}}$ & $\stackrel{\mathbb{\infty}}{\mathbb{\infty}}$ \\
\hline 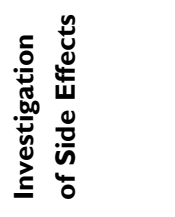 & 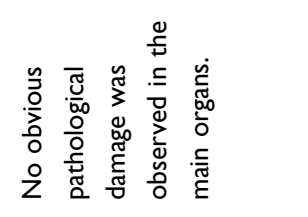 & 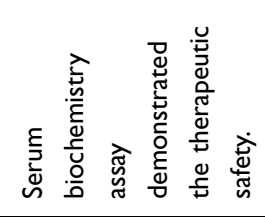 & 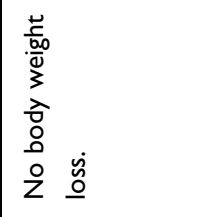 \\
\hline 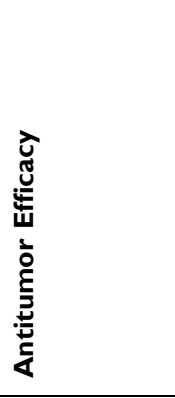 & 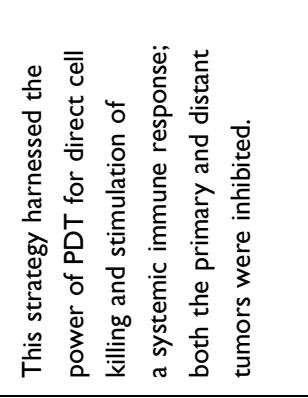 & 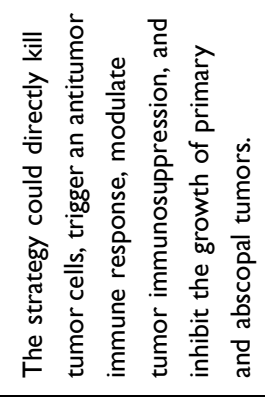 & 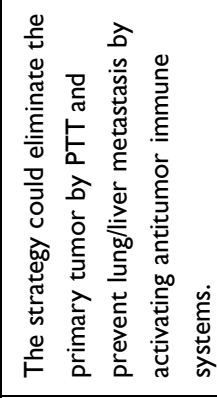 \\
\hline 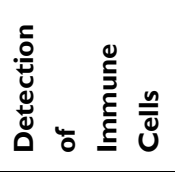 & 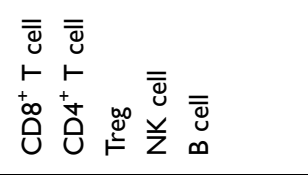 & 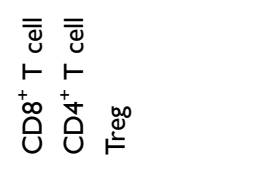 & 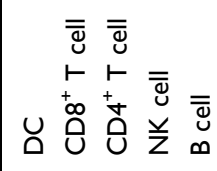 \\
\hline 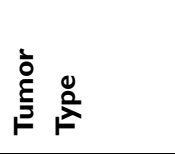 & 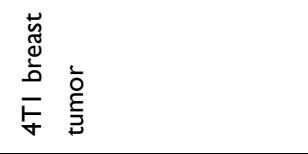 & 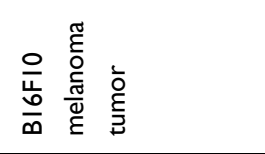 & 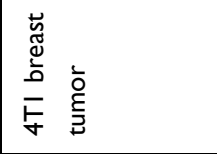 \\
\hline 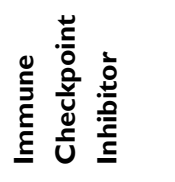 & 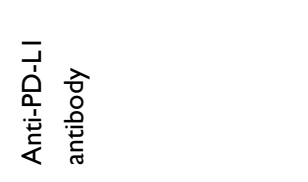 & 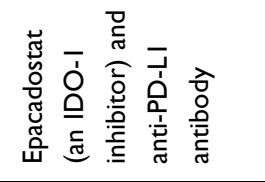 & 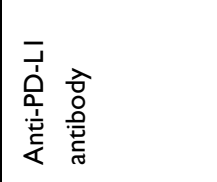 \\
\hline 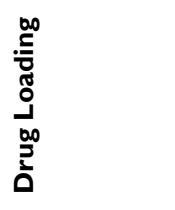 & 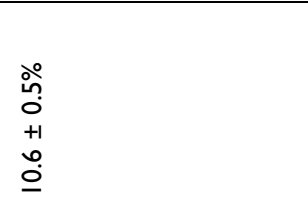 & 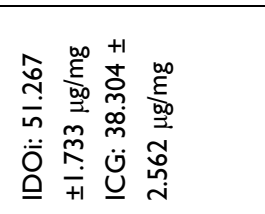 & 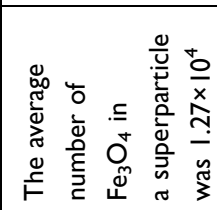 \\
\hline 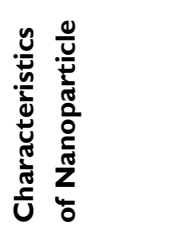 & 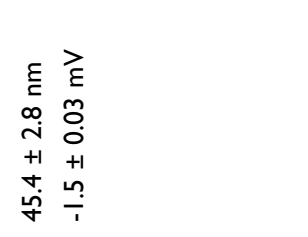 & 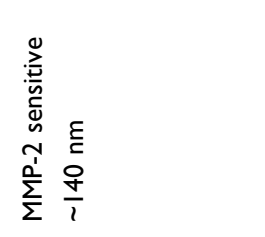 & 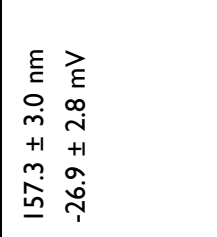 \\
\hline 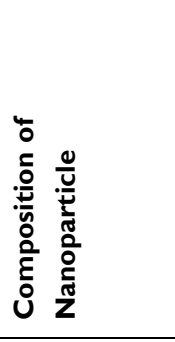 & 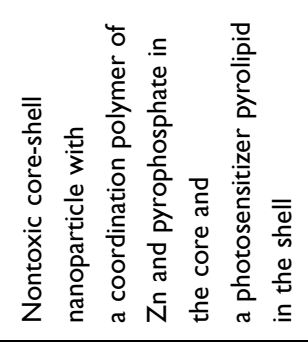 & 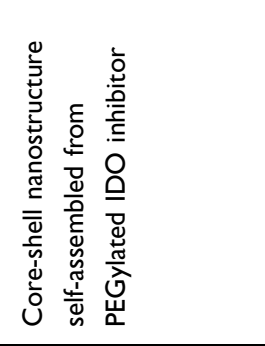 & 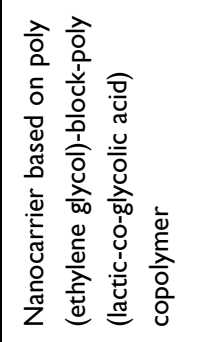 \\
\hline 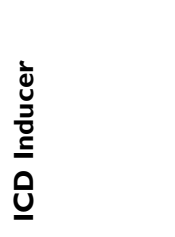 & 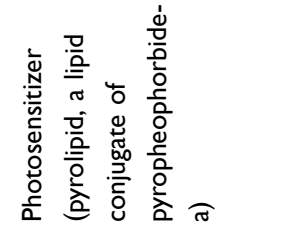 & 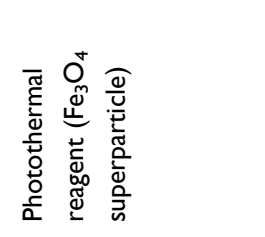 & 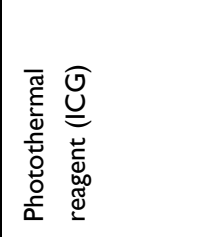 \\
\hline
\end{tabular}


was exposed to the acidic and enzymatic tumor microenvironment, the encapsulated drugs were released. The codelivery of the OXA prodrug and PPa could trigger ICD of tumor cells when irradiated with laser. The combination of prodrugvesicle-induced ICD and antibody-mediated CD47 blockade further facilitated DC maturation, promoted antigen presentation by DCs, and eventually propagated antitumor immunity. This multipronged approach of chemotherapy, PDT, and immunotherapy was demonstrated to efficiently inhibit the growth of both primary and abscopal tumors, suppress tumor metastasis, and prevent tumor recurrence (Figure 7). Recent research on combination cancer therapies using nanoparticle-based multipronged ICD inducers and immune checkpoint inhibitors is summarized in Table 3.

A
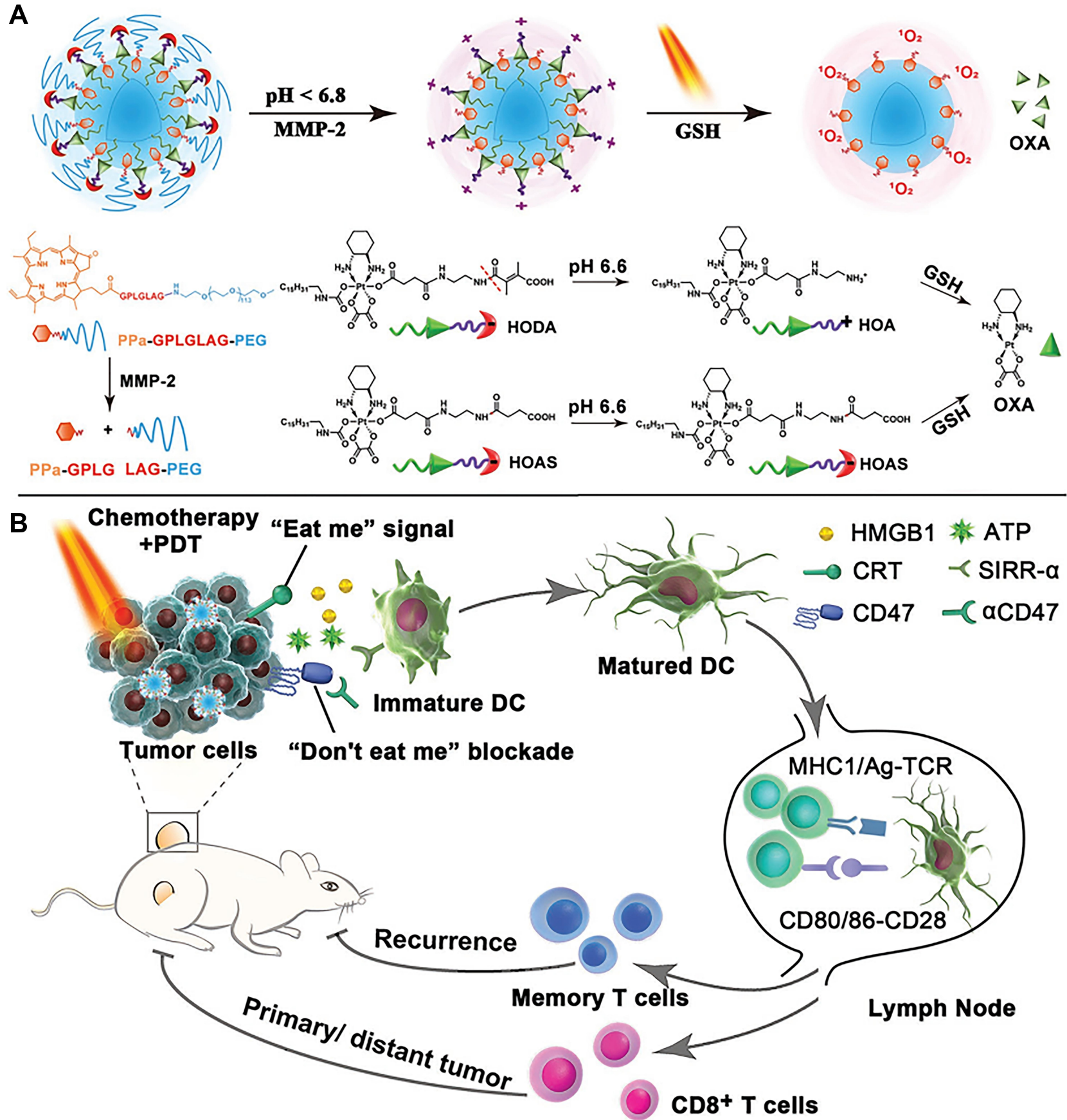

Figure 7 Schematic illustration of the prodrug vesicles for cancer immunotherapy by ICD induction and CD47 blockade. (A) Schematic design of the acidity and MMP-2 dual-responsive prodrug vesicles. (B) Simplified mechanism of prodrug vesicle -mediated chemo-immunotherapy and CD47 blockade to inhibit tumor growth, recurrence, and distant metastasis. Adapted with permission from . Copyright (C) 2019 John Wiley and Sons. ${ }^{93}$ 


\begin{tabular}{|c|c|c|c|c|}
\hline ֻّ & $\underset{\underset{\infty}{\infty}}{\sigma}$ & ஓ & $\bar{\Xi}$ & $\Xi$ \\
\hline 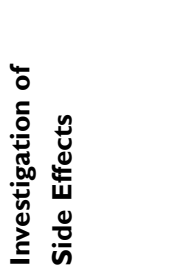 & 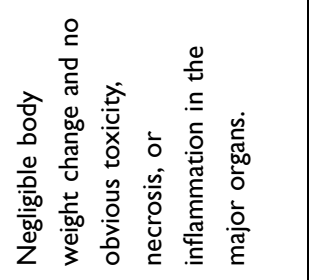 & 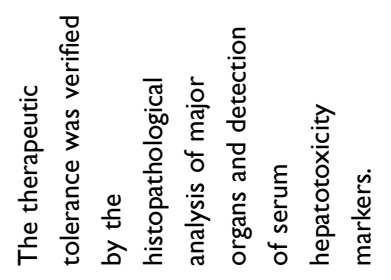 & 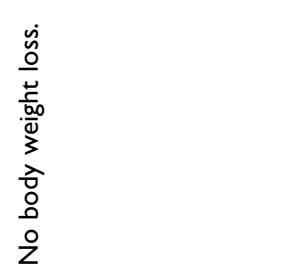 & 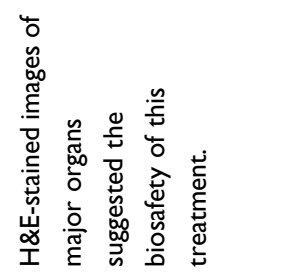 \\
\hline 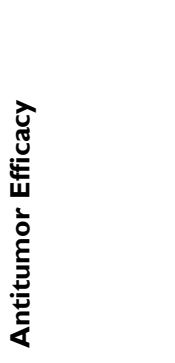 & 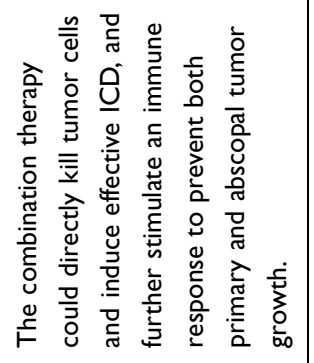 & 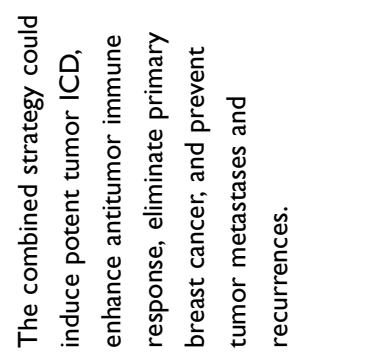 & 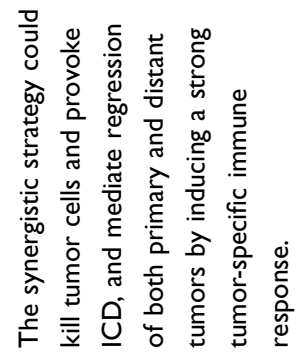 & 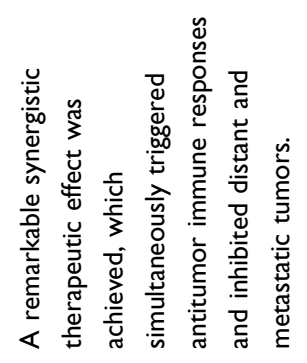 \\
\hline 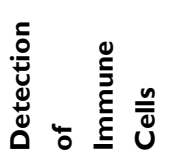 & 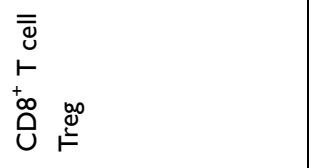 & 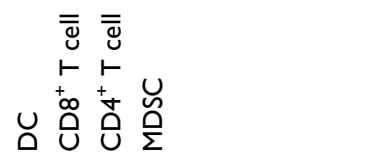 & 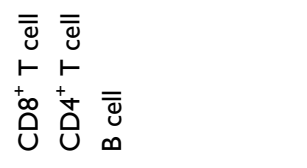 & 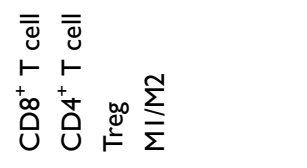 \\
\hline 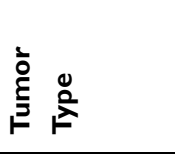 & 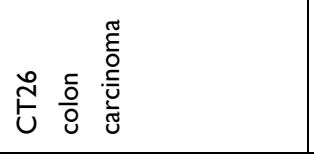 & 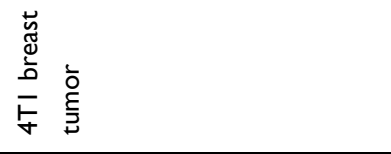 & 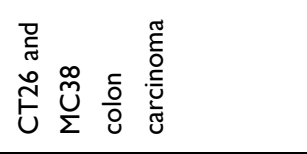 & 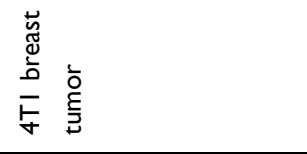 \\
\hline 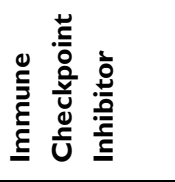 & 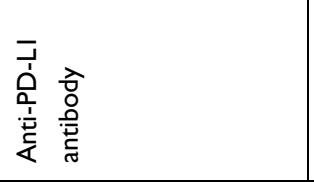 & 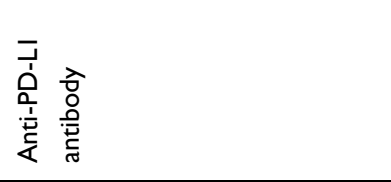 & 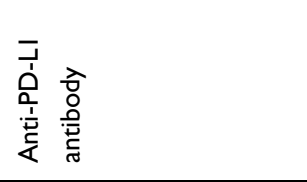 & 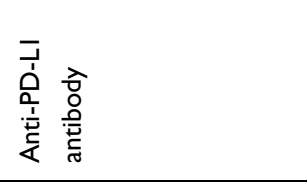 \\
\hline 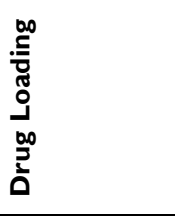 & 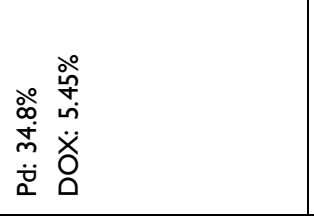 & 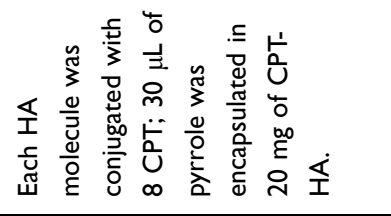 & $\begin{array}{l}\stackrel{\circ}{\circ} \\
\stackrel{i}{\grave{x}} \\
\text { Oे }\end{array}$ & 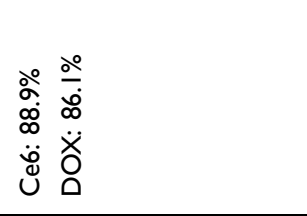 \\
\hline 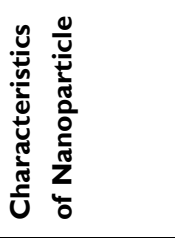 & 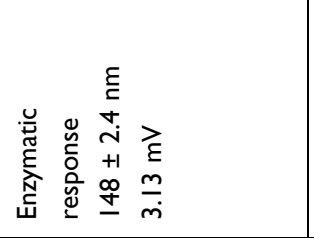 & 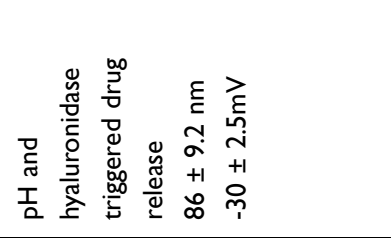 & 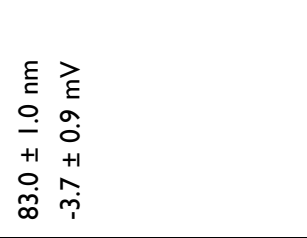 & 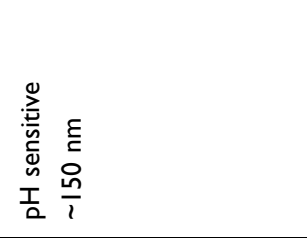 \\
\hline 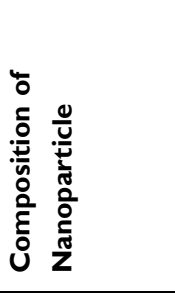 & 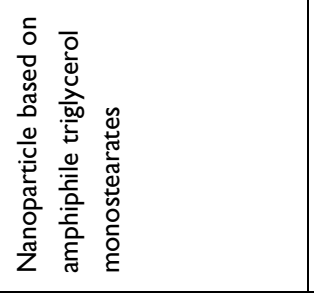 & 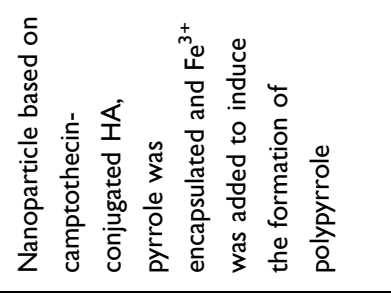 & 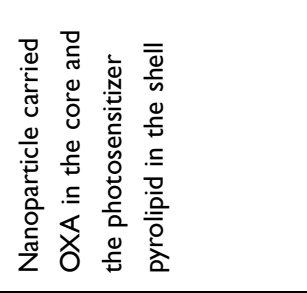 & 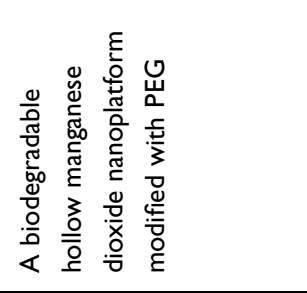 \\
\hline 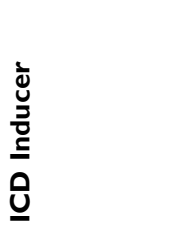 & 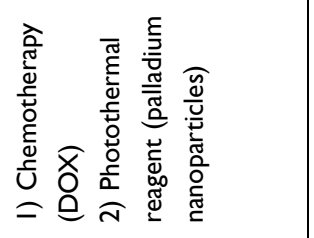 & 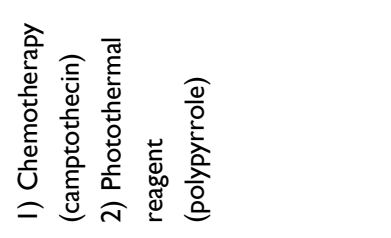 & 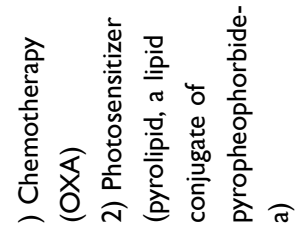 & 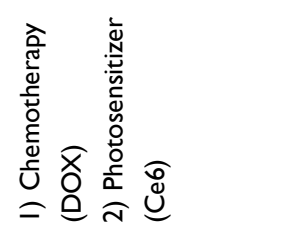 \\
\hline
\end{tabular}




\begin{tabular}{|c|c|c|c|}
\hline 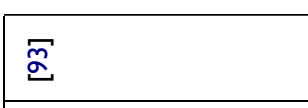 & $\Phi$ & g & 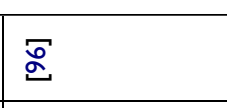 \\
\hline 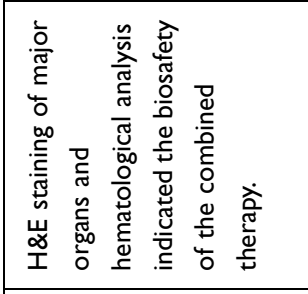 & 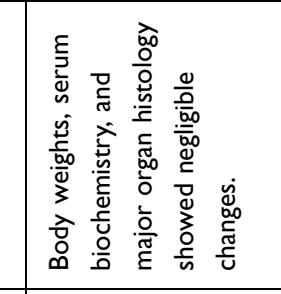 & 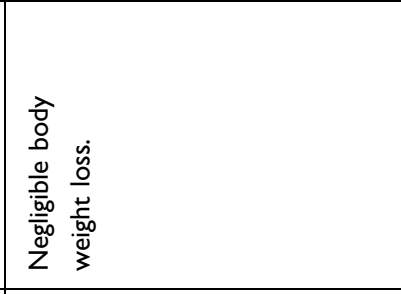 & \\
\hline 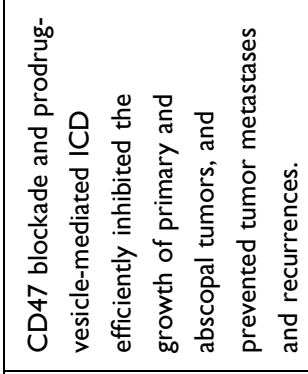 & 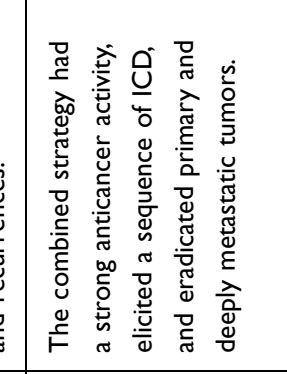 & 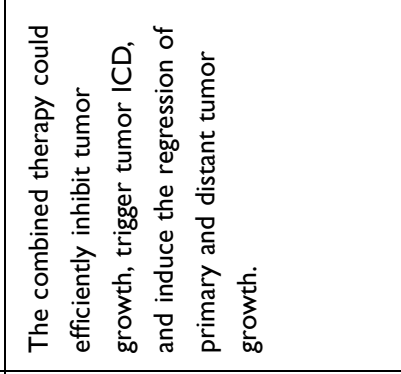 & 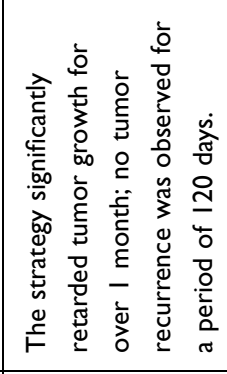 \\
\hline 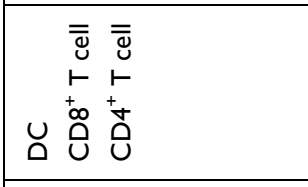 & 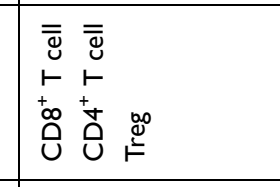 & 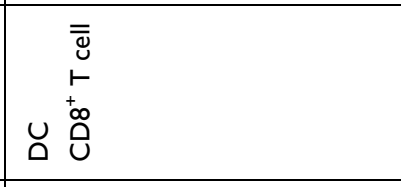 & 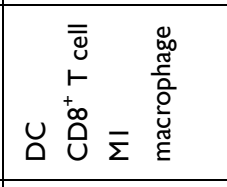 \\
\hline 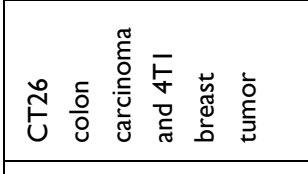 & 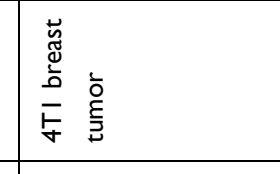 & 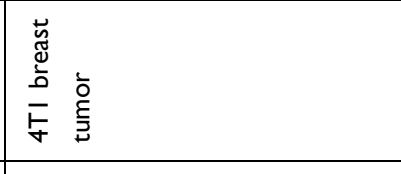 & 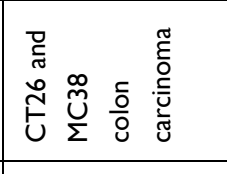 \\
\hline & 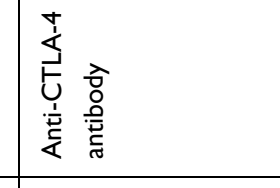 & 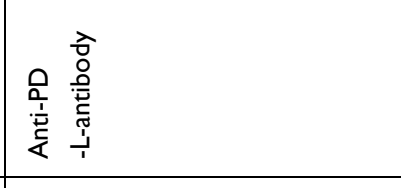 & 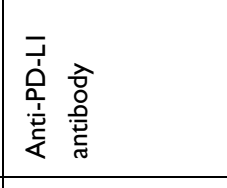 \\
\hline 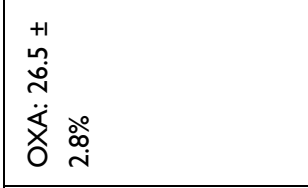 & 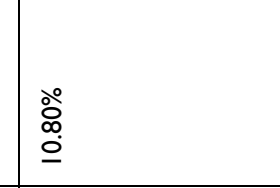 & 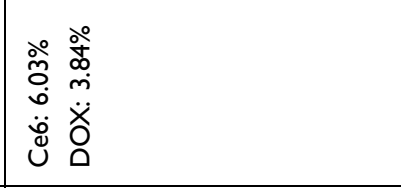 & 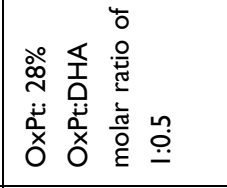 \\
\hline & 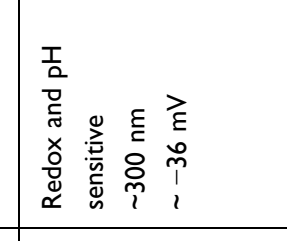 & 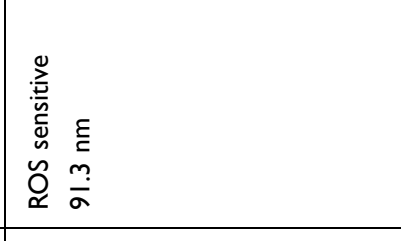 & 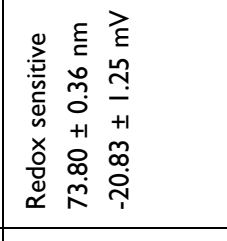 \\
\hline 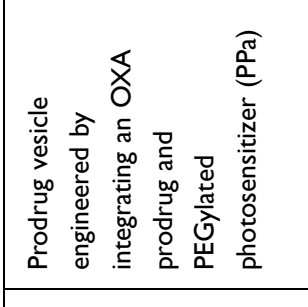 & 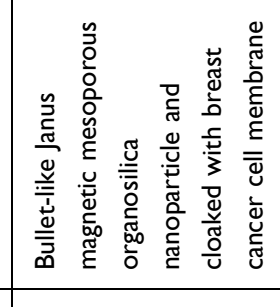 & 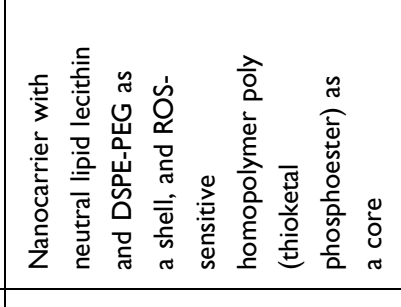 & 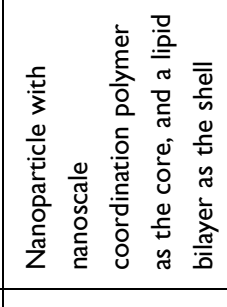 \\
\hline 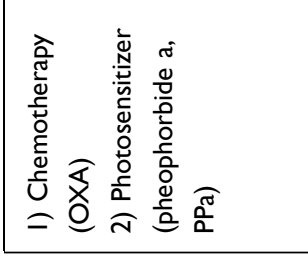 & 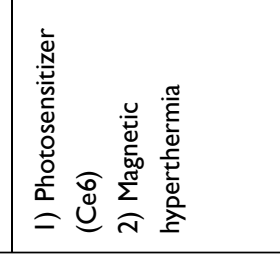 & 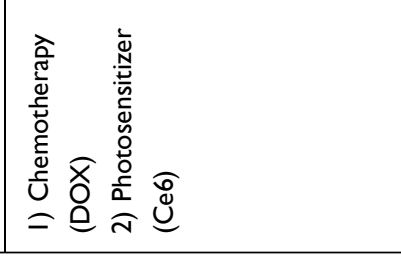 & 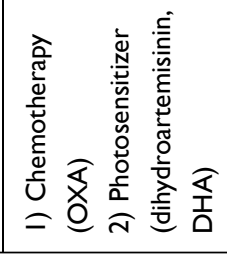 \\
\hline
\end{tabular}




\section{Conclusions and Future Challenges}

Immunotherapy has achieved exciting clinical responses and become a promising strategy to completely cure cancer. However, low response rates and potential side effects remain significant hurdles to its widespread clinical application. Importantly, the formation of an immunosuppressive tumor microenvironment is one of the most serious challenges with respect to anticancer efficacy, which is mainly induced by low tumor immunogenicity and the inactivation of tumor-killing immune cells. Therefore, ICD inducers were proposed to increase tumor immunogenicity, while immune checkpoint inhibitors were applied to relieve the suppression of immune cells. These approaches for tumor treatment have been demonstrated by various studies, especially combination strategies involving ICD inducers and immune checkpoint inhibitors. Unfortunately, the common chemical and physical methods of inducing tumor ICD may be limited by toxicity and low efficiency. As a result, nanoparticle-based chemical and physical ICD inducers are emerging, which offer an opportunity to eliminate side effects and improve the efficiency of therapeutic agents. Further combination with immune checkpoint blockade can amplify the tumor killing and immune activation effects.

Nevertheless, there are still some limitations to the use of nanoparticles in drug delivery. The most important of these is cytotoxicity, which is primarily caused by the material, size, surface charge, and concentration of nanoparticles. ${ }^{97}$ Artificial nanoparticles may be immunogenic and easily engulfed and eliminated by immune cells. ${ }^{51}$ Besides, some non-degradable nanoparticles of a specific size may be retained in the lung, liver, kidney, or other sites and cause severe damage. ${ }^{98}$ Moreover, the complexity and difficulty of the preparation of nanoparticles has also hindered their commercial and clinical applications. Emerging twodimensional nanomaterials, such as black phosphorus nanosheets, ${ }^{99-103}$ tellurium-selenium nanomaterials, ${ }^{104}$ boron nanosheets, ${ }^{105}$ antimonene nanosheets, ${ }^{106,107}$ and MXene $\left(\mathrm{Ti}_{3} \mathrm{C}_{2}\right)$-integrated cellulose hydrogels, ${ }^{108}$ as metalfree photothermal agents, have attracted increased attention in biomedical applications because they are easily biodegradable and biocompatible, in contrast to conventional inorganic nanoparticles, and provide more opportunities for use of nanoparticles in drug delivery. It worth noting that, although nanoparticle-based immunotherapy has shown excellent and promising results in preclinical studies, supporting the idea that it has enormous potential for fighting cancer, to date, few strategies have been tested in clinical trials and none of them has yet been approved for clinical use. ${ }^{50}$ However, if all these limitations are considered and addressed, the practical application of nanoparticle drug delivery systems will eventually become a reality.

In addition, tumor immunotherapy faces the challenge of low patient response rates owing to tumor immunosuppression, tumor heterogeneity, variability in cancer types and stages, and adaptive resistance. Although the emergence of immune checkpoint inhibitors is promising, the low expression of related ligands in tumors reduces the therapeutic response. Therefore, the discovery of immune checkpoint inhibitors with general applicability is of great importance. Moreover, the evaluation of immune responses after treatment is limited by the current static and invasive methods. To facilitate the discovery of immunotherapeutic agents and longitudinal evaluation of immunotherapeutic outcomes, real-time imaging of immune response is highly desirable. ${ }^{109}$

Besides, the potencies of different nanoparticle platforms or applications cannot be easily compared as each experiment was independently performed. Specifically, it is important to understand the impact of each combinatorial approach on the tumor immunosuppression microenvironment; this will be beneficial for the achievement of potent antitumor effects. As only a few stimuli have been demonstrated to induce ICD, there is an urgent need to identify more compounds or modalities that can render cell death immunogenic. New nanoparticles with appropriate properties for targeted delivery of ICD inducers are also critically needed.

\section{Acknowledgments}

This work was supported by New Century 151 Talent Project of Zhejiang Province; and National Key Research and Development Projects Intergovernmental Cooperation in Science and Technology of China [2018YFE0126900].

\section{Disclosure}

The authors report no conflicts of interest in this work.

\section{References}

1. Bray F, Ferlay J, Soerjomataram I, Siegel RL, Torre LA, Jemal A. Global cancer statistics 2018: GLOBOCAN estimates of incidence and mortality worldwide for 36 cancers in 185 countries. CA Cancer J Clin. 2018;68:394-424. doi:10.3322/caac.21492

2. Turajlic S, Swanton C. Metastasis as an evolutionary process. Science. 2016;352:169-175. doi:10.1126/science.aaf2784

3. Rosenberg SA, Restifo NP, Yang JC, Morgan RA, Dudley ME. Adoptive cell transfer: a clinical path to effective cancer immunotherapy. Nat Rev Cancer. 2008;8:299-308. doi:10.1038/ $\operatorname{nrc} 2355$ 
4. Ribas A, Wolchok JD. Cancer immunotherapy using checkpoint blockade. Science. 2018;359:1350-1355. doi:10.1126/science. aar4060

5. Alev M, Egenberger L, Mühleisen L, et al. Targeting of drug-loaded nanoparticles to tumor sites increases cell death and release of danger signals. $J$ Control Release. 2018;285:67-80. doi:10.1016/j.jconrel.2018.07.007

6. Smyth MJ, Ngiow SF, Ribas A, Teng MW. Combination cancer immunotherapies tailored to the tumour microenvironment. Nat Rev Clin Oncol. 2016;13:143-158. doi:10.1038/ nrclinonc.2015.209

7. Marçais A, Walzer T. An immunosuppressive pathway for tumor progression. Nat Med. 2018;24:260-261. doi:10.1038/nm.4508

8. Ruella M, Klichinsky M, Kenderian SS, et al. Overcoming the immunosuppressive tumor microenvironment of Hodgkin lymphoma using chimeric antigen receptor T cells. Cancer Discov. 2017;7:1154-1167. doi:10.1158/2159-8290.CD-16-0850

9. Zhou J, Wang G, Chen Y, Wang H, Hua Y, Cai Z. Immunogenic cell death in cancer therapy: present and emerging inducers. J Cell Mol Med. 2019;23:4854 4865. doi:10.1111/jcmm.14356

10. Kurtulus S, Madi A, Escobar G, et al. Checkpoint blockade immunotherapy induces dynamic changes in PD-1(-)CD8(+) tumor-infiltrating T cells. Immunity. 2019;50:181-194.e186. doi:10.1016/j.immuni.2018.11.014

11. Pfannenstiel LW, Diaz-Montero CM, Tian YF, Scharpf J, Ko JS, Gastman BR. Immune-checkpoint blockade opposes CD8(+) T-cell suppression in human and murine cancer. Cancer Immunol Res. 2019;7:510-525. doi:10.1158/2326-6066.CIR-180054

12. Xiao Y, Freeman GJ. The microsatellite instable subset of colorectal cancer is a particularly good candidate for checkpoint blockade immunotherapy. Cancer Discov. 2015;5:16-18. doi:10.1158/ 2159-8290.CD-14-1397

13. Chen Q, Liu L, Lu Y, et al. Tumor microenvironment-triggered aggregated magnetic nanoparticles for reinforced image-guided immunogenic chemotherapy. Adv Sci (Weinh). 2019;6:1802134. doi:10.1002/advs.201802134

14. Chen $\mathrm{C}$, Ni X, Jia S, et al. Massively evoking immunogenic cell death by focused mitochondrial oxidative stress using an AIE luminogen with a twisted molecular structure. Adv Mater. 2019;31:e1904914. doi:10.1002/adma.201904914

15. Li J, Cui D, Huang $J$, et al. Organic semiconducting pro-nanostimulants for near-infrared photoactivatable cancer immunotherapy. Angew Chem Int Ed Engl. 2019;58:12680-12687. doi:10.1002/anie.201906288

16. Krysko DV, Garg AD, Kaczmarek A, Krysko O, Agostinis P, Vandenabeele P. Immunogenic cell death and DAMPs in cancer therapy. Nat Rev Cancer. 2012;12:860-875. doi:10.1038/nrc3380

17. Zhao X, Yang K, Zhao R, et al. Inducing enhanced immunogenic cell death with nanocarrier-based drug delivery systems for pancreatic cancer therapy. Biomaterials. 2016;102:187-197. doi:10.1016/j.biomaterials.2016.06.032

18. Fuchs Y, Steller H. Programmed cell death in animal development and disease. Cell. 2011;147:742-758. doi:10.1016/j. cell.2011.10.033

19. Rock KL, Hearn A, Chen CJ, Yan S. Natural endogenous adjuvants. Springer Semin Immunopathol. 2005;26:231-246. doi:10.1007/s00281-004-0173-3

20. Garg AD, Krysko DV, Vandenabeele P, Agostinis P. DAMPs and PDT-mediated photo-oxidative stress: exploring the unknown. Photochem Photobiol Sci. 2011;10:670-680. doi:10.1039/ c0pp00294a

21. Garg AD, Nowis D, Golab J, Vandenabeele P, Krysko DV, Agostinis P. Immunogenic cell death, DAMPs and anticancer therapeutics: an emerging amalgamation. Biochim Biophys Acta. 2010;1805:53-71. doi:10.1016/j.bbcan.2009.08.003
22. Matzinger P. Tolerance, danger, and the extended Family. Annu Rev Immunol. 1994;12:991-1045. doi:10.1146/annurev. iy.12.040194.005015

23. Obeid M, Tesniere A, Ghiringhelli F, et al. Calreticulin exposure dictates the immunogenicity of cancer cell death. Nat Med. 2007;13:54-61. doi:10.1038/nm1523

24. Garg AD, Krysko DV, Verfaillie T, et al. A novel pathway combining calreticulin exposure and ATP secretion in immunogenic cancer cell death. EMBO J. 2012;31:1062-1079. doi:10.1038/emboj.2011.497

25. Rock KL, Kono H. The inflammatory response to cell death. Annu Rev Pathol. 2008;3:99-126. doi:10.1146/annurev. pathmechdis.3.121806.151456

26. Zitvogel L, Kepp O, Kroemer G. Decoding cell death signals in inflammation and immunity. Cell. 2010;140:798-804. doi:10.1016/j.cell.2010.02.015

27. Palm NW, Medzhitov R. Pattern recognition receptors and control of adaptive immunity. Immunol Rev. 2009;227:221-233. doi:10.1111/j.1600-065X.2008.00731.x

28. Krysko DV, Agostinis P, Krysko O, et al. Emerging role of damage-associated molecular patterns derived from mitochondria in inflammation. Trends Immunol. 2011;32:157-164. doi:10.1016/ j.it.2011.01.005

29. Showalter A, Limaye A, Oyer JL, et al. Cytokines in immunogenic cell death: applications for cancer immunotherapy. Cytokine. 2017;97:123-132. doi:10.1016/j.cyto.2017.05.024

30. Afshar N, Black BE, Paschal BM. Retrotranslocation of the chaperone calreticulin from the endoplasmic reticulum lumen to the cytosol. Mol Cell Biol. 2005;25:8844-8853. doi:10.1128/ MCB.25.20.8844-8853.2005

31. Bedard K, Szabo E, Michalak M, Opas M. Cellular functions of endoplasmic reticulum chaperones calreticulin, calnexin, and ERp57. Int Rev Cytol. 2005;245:91-121.

32. Gardai SJ, McPhillips KA, Frasch SC, et al. Cell-surface calreticulin initiates clearance of viable or apoptotic cells through trans-activation of LRP on the phagocyte. Cell. 2005;123:321-334. doi:10.1016/j.cell.2005.08.032

33. Panaretakis $\mathrm{T}$, Kepp O, Brockmeier U, et al. Mechanisms of pre-apoptotic calreticulin exposure in immunogenic cell death. EMBO J. 2009;28:578-590. doi:10.1038/emboj.2009.1

34. Garg AD, Krysko DV, Vandenabeele P, Agostinis P. Hypericinbased photodynamic therapy induces surface exposure of damage-associated molecular patterns like HSP70 and calreticulin. Cancer Immunol Immunother. 2012;61:215-221. doi:10.1007/s00262-011-1184-2

35. Andersson U, Tracey KJ. HMGB1 is a therapeutic target for sterile inflammation and infection. Annu Rev Immunol. 2011;29:139-162. doi:10.1146/annurev-immunol-030409-101323

36. Scaffidi P, Misteli T, Bianchi ME. Release of chromatin protein HMGB1 by necrotic cells triggers inflammation. Nature. 2002;418:191-195. doi:10.1038/nature00858

37. Wang $\mathrm{H}$, Bloom $\mathrm{O}$, Zhang $\mathrm{M}$, et al. HMG-1 as a late mediator of endotoxin lethality in mice. Science. 1999;285:248-251. doi:10.1126/science.285.5425.248

38. Apetoh L, Ghiringhelli F, Tesniere A, et al. Toll-like receptor 4-dependent contribution of the immune system to anticancer chemotherapy and radiotherapy. Nat Med. 2007;13:1050-1059. doi:10.1038/nm1622

39. Schiraldi M, Raucci A, Munoz LM, et al. HMGB1 promotes recruitment of inflammatory cells to damaged tissues by forming a complex with CXCL12 and signaling via CXCR4. J Exp Med. 2012;209:551-563. doi:10.1084/jem.20111739

40. Apetoh L, Ghiringhelli F, Tesniere A, et al. The interaction between HMGB1 and TLR4 dictates the outcome of anticancer chemotherapy and radiotherapy. Immunol Rev. 2007;220:47-59. doi:10.1111/j.1600-065X.2007.00573.x 
41. Burnstock G. Physiology and pathophysiology of purinergic neurotransmission. Physiol Rev. 2007;87:659-797.

42. Lazarowski ER, Sesma JI, Seminario-Vidal L, Kreda SM. Molecular mechanisms of purine and pyrimidine nucleotide release. Adv Pharmacol. 2011;61:221-261.

43. Michaud M, Martins I, Sukkurwala AQ, et al. Autophagydependent anticancer immune responses induced by chemotherapeutic agents in mice. Science. 2012;334:1573-1577. doi:10.1126/science. 1208347

44. Kroemer G, Galluzzi L, Kepp O, Zitvogel L. Immunogenic cell death in cancer therapy. Annu Rev Immunol. 2013;31:51-72. doi:10.1146/annurev-immunol-032712-100008

45. Ohta A, Sitkovsky M. Role of G-protein-coupled adenosine receptors in downregulation of inflammation and protection from tissue damage. Nature. 2002;414:916-920. doi:10.1038/ $414916 a$

46. Stagg J, Beavis PA, Divisekera U, Liu MCP, Smyth MJ. CD73deficient mice are resistant to carcinogenesis. Cancer Res. 2012;72:2190-2196. doi:10.1158/0008-5472.CAN-12-0420

47. Elliott MR, Chekeni FB, Trampont PC, et al. Nucleotides released by apoptotic cells act as a find-me signal to promote phagocytic clearance. Nature. 2009;461:282-286. doi:10.1038/nature08296

48. Kronlage M, Song J, Sorokin L, et al. Autocrine purinergic receptor signaling is essential for macrophage chemotaxis. Sci Signal. 2010;3:ra55. doi:10.1126/scisignal.2000588

49. Idzko M, Hammad H, van Nimwegen M, et al. Extracellular ATP triggers and maintains asthmatic airway inflammation by activating dendritic cells. Nat Med. 2007;13:913-919. doi:10.1038/ nm1617

50. Grimaldi AM, Incoronato M, Salvatore M, Soricelli A. Nanoparticle-based strategies for cancer immunotherapy and immunodiagnostics. Nanomedicine (Lond). 2017;12:2349-2365. doi:10.2217/nnm-2017-0208

51. Dobrovolskaia MA, Aggarwal P, Hall JB, McNeil SE. Preclinical studies to understand nanoparticle interaction with the immune system and its potential effects on nanoparticle biodistribution. Mol Pharm. 2008;5:487-495. doi:10.1021/mp800032f

52. Zeng $\mathrm{Z}, \mathrm{Pu} \mathrm{K}$. Improving cancer immunotherapy by cell membrane-camouflaged nanoparticles. Adv Funct Mater. 2020;30:2004397. doi:10.1002/adfm.202004397

53. Zhang $\mathrm{C}, \mathrm{Pu} \mathrm{K}$. Molecular and nanoengineering approaches towards activatable cancer immunotherapy. Chem Soc Rev. 2020;49:4234-4253. doi:10.1039/C9CS00773C

54. Galluzzi L, Buqué A, Kepp O, Zitvogel L, Kroemer G. Immunogenic cell death in cancer and infectious disease. Nat Rev Immunol. 2016;17:97-111.

55. André T, Tournigand C, Rosmorduc O, et al. Gemcitabine combined with oxaliplatin (GEMOX) in advanced biliary tract adenocarcinoma: a GERCOR study. Ann Oncol. 2004;15:1339-1343. doi:10.1093/annonc/mdh351

56. Qi L, Chen F, Lin H, et al. Nanocarrier-mediated chemo-immuno therapy arrested cancer progression and induced tumor dormancy in desmoplastic melanoma. ACS Nano. 2018;12:7812-7825. doi:10.1021/acsnano.8b01890

57. Qi J, Li W, Lu K, et al. $\mathrm{pH}$ and thermal dual-sensitive nanoparticle-mediated synergistic antitumor effect of immunotherapy and microwave thermotherapy. Nano Lett. 2019;19:4949-4959. doi:10.1021/acs.nanolett.9b01061

58. Kuai R, Yuan W, Son S, et al. Elimination of established tumors with nanodisc-based combination chemoimmunotherapy. Sci Adv. 2018;4:eaao1736. doi:10.1126/sciadv.aao1736

59. Lu J, Liu X, Liao YP, et al. Breast cancer chemo-immunotherapy through liposomal delivery of an immunogenic cell death stimulus plus interference in the IDO-1 pathway. ACS Nano. 2018;12:11041-11061. doi:10.1021/acsnano.8b05189
60. Feng B, Zhou F, Hou B, et al. Binary cooperative prodrug nanoparticles improve immunotherapy by synergistically modulating immune tumor microenvironment. Adv Mater. 2018;30:1803001. doi:10.1002/adma.201803001

61. Chen Y, Xia R, Huang Y, et al. An immunostimulatory dual-functional nanocarrier that improves cancer immunochemotherapy. Nat Commun. 2016;7:13443. doi:10.1038/ncomms13443

62. Zhai Q, Chen Y, Xu J, et al. Lymphoma immunochemotherapy: targeted delivery of doxorubicin via a dual functional nanocarrier. Mol Pharm. 2017;14:3888-3895. doi:10.1021/acs. molpharmaceut.7b00606

63. Wan Z, Sun J, Xu J, et al. Dual functional immunostimulatory polymeric prodrug carrier with pendent indoximod for enhanced cancer immunochemotherapy. Acta Biomater. 2019;90:300-313. doi:10.1016/j.actbio.2019.03.048

64. Sun JJ, Chen YC, Huang YX, et al. Programmable co-delivery of the immune checkpoint inhibitor NLG919 and chemotherapeutic doxorubicin via a redox-responsive immunostimulatory polymeric prodrug carrier. Acta Pharmacol. 2017;38:823-834.

65. Mei KC, Liao YP, Jiang J, et al. Liposomal delivery of mitoxantrone and a cholesteryl indoximod prodrug provides effective chemo-immunotherapy in multiple solid tumors. ACS Nano. 2020;14:13343-13366. doi:10.1021/acsnano.0c05194

66. Sharma RA, Plummer R, Stock JK, et al. Clinical development of new drug-radiotherapy combinations. Nat Rev Clin Oncol. 2016;13:627-642. doi:10.1038/nrclinonc.2016.79

67. Ludgate CM. Optimizing cancer treatments to induce an acute immune response: radiation abscopal effects, PAMPs, and DAMPs. Clin Cancer Res. 2012;18:4522-4525. doi:10.1158/ 1078-0432.CCR-12-1175

68. Cheng L, Wang C, Feng L, Yang K, Liu Z. Functional nanomaterials for phototherapies of cancer. Chem Rev. 2014;114:10869-10939. doi:10.1021/cr400532z

69. Bown S. Phototherapy of tumors. World J Surg. 1983;7:700-709. doi:10.1007/BF01655209

70. Xu J, Xu L, Wang C, et al. Near-infrared-triggered photodynamic therapy with multitasking upconversion nanoparticles in combination with checkpoint blockade for immunotherapy of colorectal cancer. ACS Nano. 2017;11:4463-4474.

71. Dolmans DE, Fukumura D, Jain RK. Photodynamic therapy for cancer. Nat Rev Cancer. 3003;3:380-387. doi:10.1038/nrc1071

72. Xie Z, Fan T, An J, et al. Emerging combination strategies with phototherapy in cancer nanomedicine. Chem Soc Rev. 2020;49:8065-8087. doi:10.1039/D0CS00215A

73. Chen J, Fan T, Xie Z, et al. Advances in nanomaterials for photodynamic therapy applications: status and challenges. Biomaterials. 2020;237:119827.

74. Vanpouille-Box C, Lacoeuille F, Belloche C, et al. Tumor eradication in rat glioma and bypass of immunosuppressive barriers using internal radiation with (188)Re-lipid nanocapsules. Biomaterials. 2011;32:6781-6790. doi:10.1016/j. biomaterials.2011.05.067

75. Zhang F, Lu G, Wen X, et al. Magnetic nanoparticles coated with polyphenols for spatio-temporally controlled cancer photothermal/immunotherapy. $J$ Control Release. 2020;326:131-139. doi:10.1016/j.jconrel.2020.06.015

76. Chen Z, Liu L, Liang R, et al. Bioinspired hybrid protein oxygen nanocarrier amplified photodynamic therapy for eliciting anti-tumor immunity and abscopal effect. ACS Nano. 2018;12:8633-8645. doi:10.1021/acsnano.8b04371

77. Wang $\mathrm{T}$, Zhang $\mathrm{H}$, Han $\mathrm{Y}$, et al. Light-enhanced $\mathrm{O}_{2}$-evolving nanoparticles boost photodynamic therapy to elicit antitumor immunity. ACS Appl Mater Interfaces. 2019;11:16367-16379. doi:10.1021/acsami.9b03541 
78. Li W, Yang J, Luo L, et al. Targeting photodynamic and photothermal therapy to the endoplasmic reticulum enhances immunogenic cancer cell death. Nat Commun. 2019;10:3349. doi:10.1038/s41467-019-11269-8

79. He H, Liu L, Liang R, et al. Tumor-targeted nanoplatform for in situ oxygenation-boosted immunogenic phototherapy of colorectal cancer. Acta Biomater. 2020;104:188-197. doi:10.1016/j. actbio.2020.01.012

80. Yu GT, Rao L, Wu H, et al. Myeloid-derived suppressor cell membrane-coated magnetic nanoparticles for cancer theranostics by inducing macrophage polarization and synergizing immunogenic cell death. Adv Funct Mater. 2018;28:1801389. doi:10.1002/adfm.201801389

81. Pu K, Li J, Luo Y. Electromagnetic nanomedicines for combinational cancer immunotherapy. Angew Chem Int Ed Engl. 2020.

82. Choi B, Choi H, Yu B, Kim DH. Synergistic local combination of radiation and anti-programmed death ligand 1 immunotherapy using radiation-responsive splintery metallic nanocarriers. ACS Nano. 2020;14:13115-13126. doi:10.1021/acsnano.0c04701

83. Yu B, Zhang W, Kwak K, Choi H, Kim DH. Electric pulse responsive magnetic nanoclusters loaded with indoleamine 2,3-dioxygenase inhibitor for synergistic immuno-ablation cancer therapy. ACS Appl Mater Interfaces. 2020;12:54415-54425. doi:10.1021/acsami.0c15679

84. Yang W, Zhang F, Deng H, et al. Smart nanovesicle-mediated immunogenic cell death through tumor microenvironment modulation for effective photodynamic immunotherapy. ACS Nano. 2020;14:620-631. doi:10.1021/acsnano.9b07212

85. Liu H, Hu Y, Sun Y, et al. Co-delivery of bee venom melittin and a photosensitizer with an organic-inorganic hybrid nanocarrier for photodynamic therapy and immunotherapy. ACS Nano. 2019;13:12638-12652. doi:10.1021/acsnano.9b04181

86. Duan X, Chan C, Guo N, Han W, Weichselbaum RR, Lin W. Photodynamic therapy mediated by nontoxic core-shell nanoparticles synergizes with immune checkpoint blockade to elicit antitumor immunity and antimetastatic effect on breast cancer. $J \mathrm{Am}$ Chem Soc. 2016;138:16686-16695. doi:10.1021/jacs.6b09538

87. Liu Y, Lu Y, Zhu X, et al. Tumor microenvironment-responsive prodrug nanoplatform via co-self-assembly of photothermal agent and IDO inhibitor for enhanced tumor penetration and cancer immunotherapy. Biomaterials. 2020;242:119933. doi:10.1016/j. biomaterials. 2020.119933

88. Ge R, Liu C, Zhang X, et al. Photothermal-activatable $\mathrm{Fe}_{(3)} \mathrm{O}_{(4)}$ superparticle nanodrug carriers with PD-L1 immune checkpoint blockade for anti-metastatic cancer immunotherapy. ACS Appl Mater Interfaces. 2018;10:20342-20355. doi:10.1021/ acsami.8b05876

89. Wen Y, Chen X, Zhu X, et al. Photothermal-chemotherapy integrated nanoparticles with tumor microenvironment response enhanced the induction of immunogenic cell death for colorectal cancer efficient treatment. ACS Appl Mater Interfaces. 2019;11:43393-43408. doi:10.1021/acsami.9b17137

90. Sun W, Du Y, Liang X, et al. Synergistic triple-combination therapy with hyaluronic acid-shelled PPy/CPT nanoparticles results in tumor regression and prevents tumor recurrence and metastasis in 4T1 breast cancer. Biomaterials. 2019;217:119264. doi:10.1016/j.biomaterials.2019.119264

91. He C, Duan X, Guo N, et al. Core-shell nanoscale coordination polymers combine chemotherapy and photodynamic therapy to potentiate checkpoint blockade cancer immunotherapy. Nat Commun. 2016;7:12499. doi:10.1038/ncomms12499

92. Yang $\mathrm{G}, \mathrm{Xu} \mathrm{L}$, Chao $\mathrm{Y}$, et al. Hollow $\mathrm{MnO}_{(2)}$ as a tumor-microenvironment-responsive biodegradable nano-platform for combination therapy favoring antitumor immune responses. Nat Commun. 2017;8:902. doi:10.1038/ s41467-017-01050-0
93. Zhou F, Feng B, Yu H, et al. Tumor microenvironment-activatable prodrug vesicles for nanoenabled cancer chemoimmunotherapy combining immunogenic cell death induction and CD47 blockade. Adv Mater. 2019;31:e1805888. doi:10.1002/adma.201805888

94. Wang Z, Zhang F, Shao D, et al. Janus nanobullets combine photodynamic therapy and magnetic hyperthermia to potentiate synergetic anti-metastatic immunotherapy. Adv Sci (Weinh). 2019;6:1901690. doi:10.1002/advs.201901690

95. Hu L, Cao Z, Ma L, et al. The potentiated checkpoint blockade immunotherapy by ROS-responsive nanocarrier-mediated cascade chemo-photodynamic therapy. Biomaterials. 2019;223:119469. doi:10.1016/j.biomaterials.2019.119469

96. Duan X, Chan C, Han W, Guo N, Weichselbaum RR, Lin W. Immunostimulatory nanomedicines synergize with checkpoint blockade immunotherapy to eradicate colorectal tumors. Nat Commun. 2019;10:1899. doi:10.1038/s41467-019-09221-x

97. Labouta HI, Asgarian N, Rinker K, Cramb DT. Meta-analysis of nanoparticle cytotoxicity via data-mining the literature. ACS Nano. 2019;13:1583-1594. doi:10.1021/acsnano.8b07562

98. Hoshyar N, Gray S, Han H, Bao G. The effect of nanoparticle size on in vivo pharmacokinetics and cellular interaction. Nanomedicine (Lond). 2016;11:673-692. doi:10.2217/nnm.16.5

99. Xie Z, Peng M, Lu R, et al. Black phosphorus-based photothermal therapy with aCD47-mediated immune checkpoint blockade for enhanced cancer immunotherapy. Light Sci Appl. 2020;9:161. doi:10.1038/s41377-020-00388-3

100. Xing C, Chen S, Qiu M, et al. Conceptually novel black phosphorus/cellulose hydrogels as promising photothermal agents for effective cancer therapy. Adv Healthc Mater. 2018;7:e1701510. doi:10.1002/adhm.201701510

101. Qiu M, Singh A, Wang D, et al. Biocompatible and biodegradable inorganic nanostructures for nanomedicine: silicon and black phosphorus. Nano Today. 2019;25:135-155. doi:10.1016/j. nantod.2019.02.012

102. Luo M, Fan T, Zhou Y, Zhang H, Mei L. 2D Black PhosphorusBased Biomedical Applications. Adv Func Mater. 2019;29 (13):1808306. doi:10.1002/adfm.201808306

103. Tang Z, Kong N, Ouyang J, et al. Phosphorus science-oriented design and synthesis of multifunctional nanomaterials for biomedical applications. Matter. 2020;2:297-322. doi:10.1016/j. matt.2019.12.007

104. Chen S, Xing C, Huang D, et al. Eradication of tumor growth by delivering novel photothermal selenium-coated tellurium nanoheterojunctions. Sci $A d v$. 2020;6:eaay6825. doi:10.1126/ sciadv.aay6825

105. Ji X, Kong N, Wang J, et al. A novel top-down synthesis of ultrathin 2D boron nanosheets for multimodal imaging-guided cancer therapy. Adv Mater. 2018;30:1803031. doi:10.1002/ adma.201803031

106. Tao W, Ji X, Zhu X, et al. Two-dimensional antimonene-based photonic nanomedicine for cancer theranostics. Adv Mater. 2018;30:e1802061. doi:10.1002/adma.201802061

107. Tao W, Ji X, Xu X, et al. Antimonene quantum dots: synthesis and application as near-infrared photothermal agents for effective cancer therapy. Angew Chem Int Ed Engl. 2017;56:11896-11900. doi:10.1002/anie.201703657

108. Xing $\mathrm{C}$, Chen $\mathrm{S}$, Liang $\mathrm{X}$, et al. Two-dimensional MXene $\left(\mathrm{Ti}_{3} \mathrm{C}_{2}\right)$ -integrated cellulose hydrogels: toward smart three-dimensional network nanoplatforms exhibiting light-induced swelling and bimodal photothermal/chemotherapy anticancer activity. ACS Appl Mater Interfaces. 2018;10:27631-27643. doi:10.1021/ acsami.8b08314

109. He S, Li J, Lyu Y, Huang J, Pu K. Near-infrared fluorescent macromolecular reporters for real-time imaging and urinalysis of cancer immunotherapy. $J$ Am Chem Soc. 2020;142:7075-7082. doi:10.1021/jacs.0c00659 


\section{Publish your work in this journal}

The International Journal of Nanomedicine is an international, peerreviewed journal focusing on the application of nanotechnology in diagnostics, therapeutics, and drug delivery systems throughout the biomedical field. This journal is indexed on PubMed Central, MedLine, CAS, SciSearch ${ }^{\mathbb{R}}$, Current Contents ${ }^{\mathbb{R}} /$ Clinical Medicine, $^{2}$
Journal Citation Reports/Science Edition, EMBase, Scopus and the Elsevier Bibliographic databases. The manuscript management system is completely online and includes a very quick and fair peer-review system, which is all easy to use. Visit http://www.dovepress.com/ testimonials.php to read real quotes from published authors. 